SUPPORTING INFORMATION

\title{
Ortho-Effect in the Bergman Cyclization: Comparison of Experimental Approaches and Dissection of Cycloaromatization Kinetics
}

\author{
Tarek A. Zeidan, Serguei V. Kovalenko, Mariappan Manoharan and Igor V. Alabugin* \\ ${ }^{\dagger}$ Department of Chemistry and Biochemistry, Florida State University, \\ Tallahassee, Florida 32306-4390 \\ E-mail: alabugin@chem.fsu.,edu \\ Tel: (850) 644-5795 \\ Fax: (850) 644-8281
}

TABLE OF CONTENTS

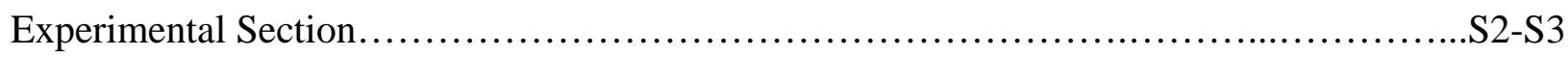

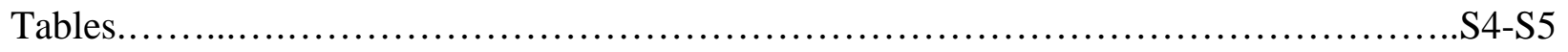

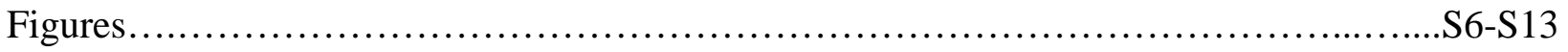

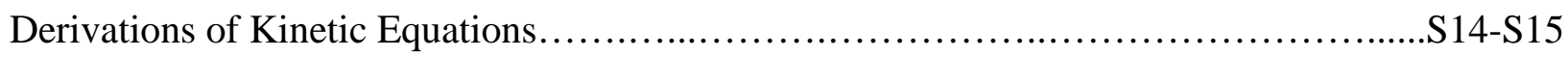

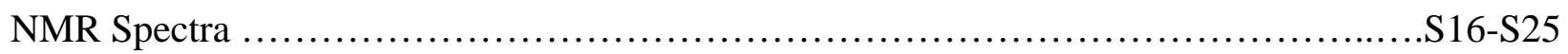

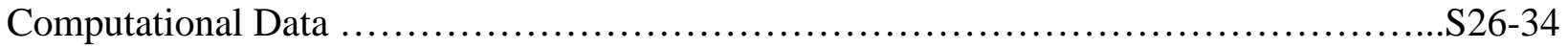




\section{EXPERIMENTAL SECTION}

General Procedure. A $10 \mathrm{ml}$ volumetric flask was charged with the enediyne, 1,2,3,4tetraphenylnaphthalene as an internal standard and 1,4-cyclohexadiene. The volumetric flask was filled to the mark with chlorobenzene. For each temperature, fifteen capillary melting-point tubes were filled with $75 \mu \mathrm{L}$ of this solution. The capillary tubes were frozen by liquid nitrogen, degassed under high vacuum, and sealed with only enough space for liquid expansion to eliminate "dead volume”. The capillary tubes were placed in preheated oil bath and removed at different time intervals. The reaction was monitored at 2-3 half-lives. Kinetic experiments were performed at four different temperatures.

For every enediyne a calibration curve was constructed and used to determine the response of enediynes, naphthalene products and the internal standard at different ratios using GC and HPLC. All curves showed linear responses to the decrease in concentrations of enediynes and the increase in concentrations of naphthalene products, under constant concentration of standard (see SI). Samples were injected into the GC or HPLC using an autosampler with the corresponding time program. The areas of the peaks corresponding to enediynes and naphthalene products were normalized against the areas of the internal standard. Concentrations of the enediynes and naphthalenes were determined from the linear calibration plots. Concentrations of enediynes were plotted versus time, and the resulting curve was fitted to a single exponential decay rate equation (Eq. 1) using Oakdale Engineering DataFit program. For the formation of products, concentration values used in rate constant determinations were corrected to the yield of the product and fitted to Eq. 2. Activation energies were determined from Arrhenius plots (lnk vs $1 / \mathrm{T})$. 
Kinetic Analysis using HPLC. The reaction mixtures were diluted with hexane before being injected into the HPLC column. The areas of the starting material or products were normalized against the areas of the internal standard. Concentrations of the enediynes or naphthalenes were determined from a linear calibration plot. Concentrations of enediynes were plotted against time, and the resulting curve was fitted to a single-exponential-decay rate equation by a Microsoft Excel program. Eq. 2 was used to fit the naphthalene data. Activation energies were determined from Arrhenius plots.

\section{Kinetic Studies under Different Concentrations of 1,4-Cyclohexadiene}

A $10 \mathrm{ml}$ volumetric flask was charged with enediyne and 1,2,3,4-tetraphenylnaphthalene or antharcene. The volumetric flask was filled to the mark with chlorobenzene. Five master solutions were prepared by taking $1 \mathrm{ml}$ from the initial stock solution and diluted to $5 \mathrm{ml}$ in a volumetric flask after adding the desired volume of 1,4-cyclohexadiene. The solutions were mixed and analyzed with HPLC and GC to determine the enediynes initial concentration. For each concentration of 1,4-cyclohexadiene, fifteen capillary melting-point tubes were filled with $75 \mu \mathrm{L}$ of the master solution. The capillary tubes were frozen by liquid nitrogen, degassed under high vacuum, and sealed with only enough space for liquid expansion. The capillary tubes were placed in an oil bath heated at the desired temperature and monitored for 2-3 half-lives. 
Table S1. Effective Rate Constants $\left(k_{e f f}, \mathrm{~s}^{-1}\right)$ for the Disappearance of Benzannelated enediynes and the Appearance of Corresponding Naphthalenes at Different Temperatures. ${ }^{a}$

\begin{tabular}{|c|c|c|}
\hline Temperature, ${ }^{\circ} \mathrm{C}$ & $k_{e f f}^{E D} \times 10^{4}$ & $k_{\text {eff }}^{\text {Napht }} \times 10^{4}$ \\
\hline \multicolumn{3}{|l|}{ 1,2-Diethynylbenzene $e^{b}$} \\
\hline $180 \pm 0.2$ & $7.83 \pm 0.36$ & $7.31 \pm 0.45$ \\
\hline $170 \pm 0.2$ & $4.50 \pm 0.15$ & $4.26 \pm 0.04$ \\
\hline $160 \pm 0.1$ & $2.27 \pm 0.06$ & $2.21 \pm 0.04$ \\
\hline $150 \pm 0.1$ & $1.02 \pm 0.10$ & $0.94 \pm 0.03$ \\
\hline \multicolumn{3}{|c|}{ 2,3-Diethynyl-1-nitrobenzene (ortho- $\left.\mathrm{NO}_{2}\right)^{c}$} \\
\hline $164 \pm 0.1$ & $24.95 \pm 0.31$ & $21.96 \pm 0.29$ \\
\hline $156 \pm 0.1$ & $16.02 \pm 0.34$ & $15.30 \pm 0.26$ \\
\hline $148 \pm 0.1$ & $9.88 \pm 0.26$ & $9.07 \pm 0.11$ \\
\hline $140 \pm 0.1$ & $5.82 \pm 0.06$ & $5.48 \pm 0.01$ \\
\hline \multicolumn{3}{|c|}{ 3,4-Diethynyl-1-nitrobenzene $\left(\text { para- } \mathrm{NO}_{2}\right)^{d}$} \\
\hline $170 \pm 0.2$ & $10.58 \pm 0.21$ & $9.64 \pm 0.12$ \\
\hline $160 \pm 0.1$ & $5.89 \pm 0.11$ & $4.99 \pm 0.05$ \\
\hline $150 \pm 0.1$ & $3.01 \pm 0.05$ & $2.67 \pm 0.03$ \\
\hline $140 \pm 0.1$ & $1.49 \pm 0.02$ & $1.26 \pm 0.01$ \\
\hline \multicolumn{3}{|c|}{ 2,3-Diethynyl-1-formylbenzene (ortho-CHO) ${ }^{e}$} \\
\hline $180 \pm 0.2$ & $33.64 \pm 1.99$ & $40.25 \pm 3.71$ \\
\hline $170 \pm 0.2$ & $20.15 \pm 0.34$ & $20.64 \pm 1.08$ \\
\hline $160 \pm 0.1$ & $11.26 \pm 0.31$ & $10.51 \pm 0.19$ \\
\hline $150 \pm 0.1$ & $5.96 \pm 0.22$ & $6.65 \pm 0.17$ \\
\hline $140 \pm 0.1$ & $3.15 \pm 0.04$ & $3.48 \pm 0.03$ \\
\hline
\end{tabular}


Table S2. Effective Rate Constants for Disappearance of Nitroenediynes ( $\left.k_{\text {eff }}^{E D}\right)$ and Appearance of Nitronaphthalenes ( $k_{\text {eff }}^{\text {Napht }}$ )at Different Temperatues. ${ }^{a}$

\begin{tabular}{|c|c|c|c|c|}
\hline \multirow[b]{2}{*}{ [1,4-CHD], M } & \multicolumn{2}{|c|}{ ortho- $\mathrm{NO}_{2}$ Enediynes ${ }^{b}$} & \multicolumn{2}{|c|}{ para- $\mathrm{NO}_{2}$ Enediynes ${ }^{c}$} \\
\hline & $k_{e f f}^{E D} \times 10^{4}$ & $k_{\text {eff }}^{\text {Napht }} \times 10^{4}$ & $k_{e f f}^{E D} \times 10^{4}$ & $k_{e f f}^{N a p h t} \times 10^{4}$ \\
\hline & \multicolumn{2}{|c|}{$T=148^{\circ} \mathrm{C}$} & \multicolumn{2}{|c|}{$T=150^{\circ} \mathrm{C}$} \\
\hline 0.73 & - & - & $3.70 \pm 0.11$ & $3.67 \pm 0.09$ \\
\hline 0.58 & $11.53 \pm 0.35$ & $11.50 \pm 0.10$ & $3.49 \pm 0.04$ & $3.12 \pm 0.02$ \\
\hline 0.44 & $10.69 \pm 0.14$ & $11.13 \pm 0.08$ & $3.26 \pm 0.07$ & $3.08 \pm 0.03$ \\
\hline 0.29 & $9.43 \pm 0.26$ & $9.07 \pm 0.11$ & $3.01 \pm 0.05$ & $2.81 \pm 0.03$ \\
\hline 0.15 & $7.00 \pm 0.03$ & $6.99 \pm 0.05$ & $2.40 \pm 0.04$ & $2.37 \pm 0.03$ \\
\hline 0.07 & $5.09 \pm 0.09$ & $5.03 \pm 0.05$ & $1.92 \pm 0.03$ & $1.47 \pm 0.02$ \\
\hline \multirow[t]{2}{*}{0.03} & $3.59 \pm 0.02$ & $3.30 \pm 0.03$ & $1.36 \pm 0.01$ & $1.29 \pm 0.02$ \\
\hline & \multicolumn{2}{|c|}{$T=156^{\circ} \mathrm{C}$} & \multicolumn{2}{|c|}{$T=160^{\circ} \mathrm{C}$} \\
\hline 0.73 & - & - & $7.56 \pm 0.14$ & $6.73 \pm 0.05$ \\
\hline 0.58 & $20.14 \pm 0.13$ & $20.13 \pm 0.24$ & $7.09 \pm 0.15$ & $6.15 \pm 0.08$ \\
\hline 0.44 & $17.43 \pm 0.23$ & $17.20 \pm 0.22$ & $6.37 \pm 0.01$ & $6.20 \pm 0.07$ \\
\hline 0.29 & $15.37 \pm 0.34$ & $15.30 \pm 0.26$ & $5.86 \pm 0.11$ & $5.68 \pm 0.05$ \\
\hline 0.15 & $11.01 \pm 0.10$ & $11.04 \pm 0.12$ & $4.59 \pm 0.08$ & $4.51 \pm 0.03$ \\
\hline 0.07 & $7.21 \pm 0.12$ & $6.17 \pm 0.09$ & $3.58 \pm 0.06$ & $3.59 \pm 0.04$ \\
\hline \multirow[t]{2}{*}{0.03} & $5.31 \pm 0.07$ & $5.90 \pm 0.14$ & $2.66 \pm 0.04$ & $2.59 \pm 0.07$ \\
\hline & \multicolumn{2}{|c|}{$T=164^{\circ} \mathrm{C}$} & \multicolumn{2}{|c|}{$T=170^{\circ} \mathrm{C}$} \\
\hline 0.73 & - & - & $14.05 \pm 0.17$ & $13.24 \pm 0.18$ \\
\hline 0.58 & $32.50 \pm 0.25$ & $32.94 \pm 0.43$ & $13.11 \pm 0.20$ & $12.59 \pm 0.08$ \\
\hline 0.44 & $27.46 \pm 0.19$ & $27.41 \pm 0.17$ & $11.84 \pm 0.21$ & $10.33 \pm 0.10$ \\
\hline 0.29 & $24.19 \pm 0.31$ & $21.96 \pm 0.29$ & $10.57 \pm 0.21$ & $9.35 \pm 0.13$ \\
\hline 0.15 & $17.01 \pm 0.12$ & $15.85 \pm 0.15$ & $8.05 \pm 0.16$ & $8.02 \pm 0.05$ \\
\hline 0.07 & $11.75 \pm 0.04$ & $11.84 \pm 0.13$ & $6.08 \pm 0.12$ & $5.80 \pm 0.03$ \\
\hline 0.03 & $8.10 \pm 0.04$ & $7.84 \pm 0.08$ & $4.47 \pm 0.05$ & $3.74 \pm 0.10$ \\
\hline
\end{tabular}

\footnotetext{
${ }^{a}$ Pseudofirst order effective rate constants $\left(k_{e f f}\right)$ are in $\mathrm{s}^{-1} .{ }^{b}$ Initial concentration of enediynes $=2.7 \times 10^{-3} \mathrm{M}$.
} ${ }^{c}$ Initial concentration of enediynes $=3.8 \times 10^{-3} \mathrm{M}$. 
(a)

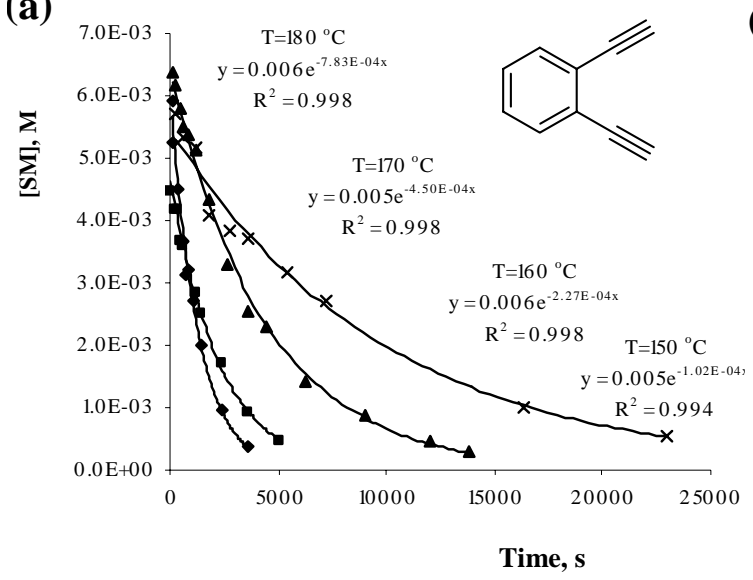

(b)

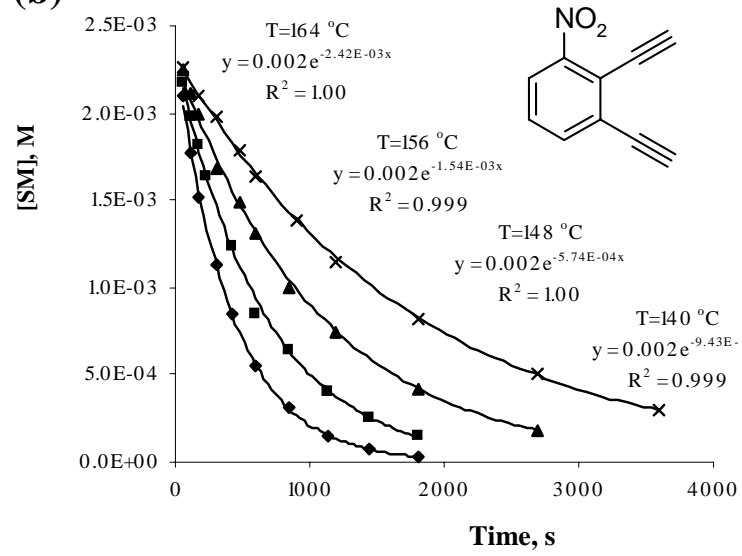

(a')

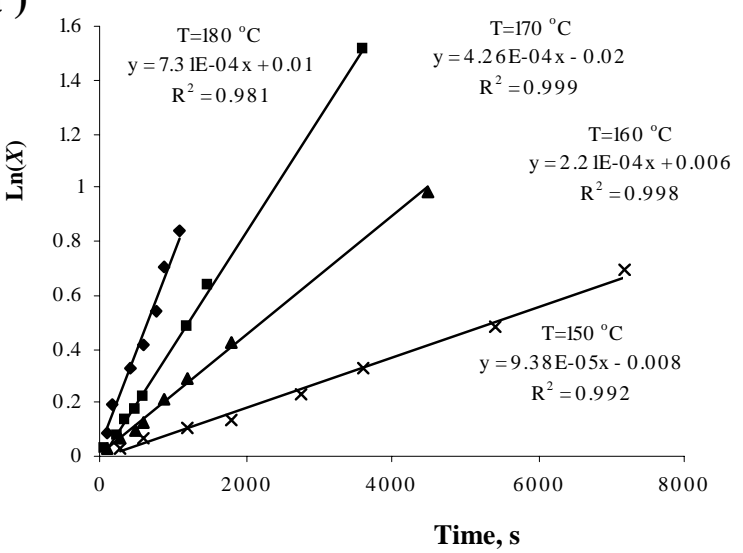

(b')

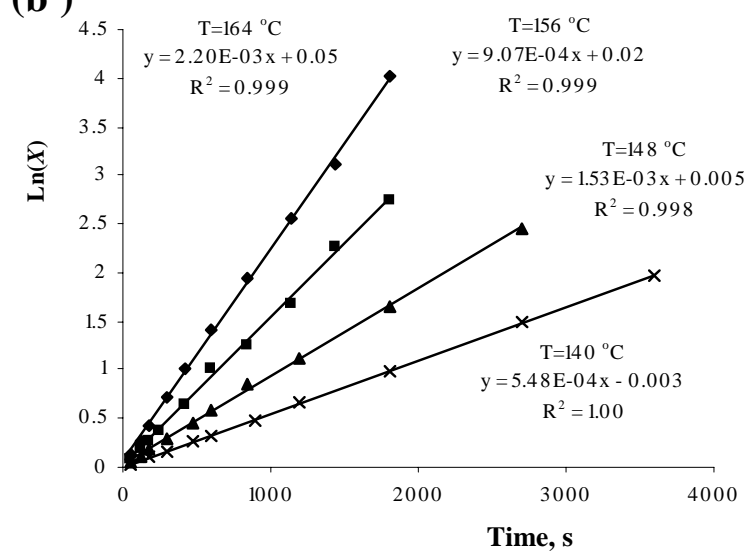

Figure S1. First-order rate constants at different temperatures determined for the disappearance of ortho-substituted enediynes: (a) 1,2-diethynylbenzene, (b) 2,3-diethynylnitrobenzene, (c) 3,4diethynylnitrobenzene, (d) 2,3-diethynylbenzaldehyde; the appearance of the corresponding naphthalene products: (a') naphthalene, (b') 1-nitronaphthalene, (c') 2-nitronaphthalene, (d') 1naphthaldehyde. ${ }^{1} X$ is defined in Eq. 2 in the manuscript.

\footnotetext{
${ }^{1}$ Kinetic analysis were performed using GC analysis except b, b’, $d$ and d' determined by HPLC
} 

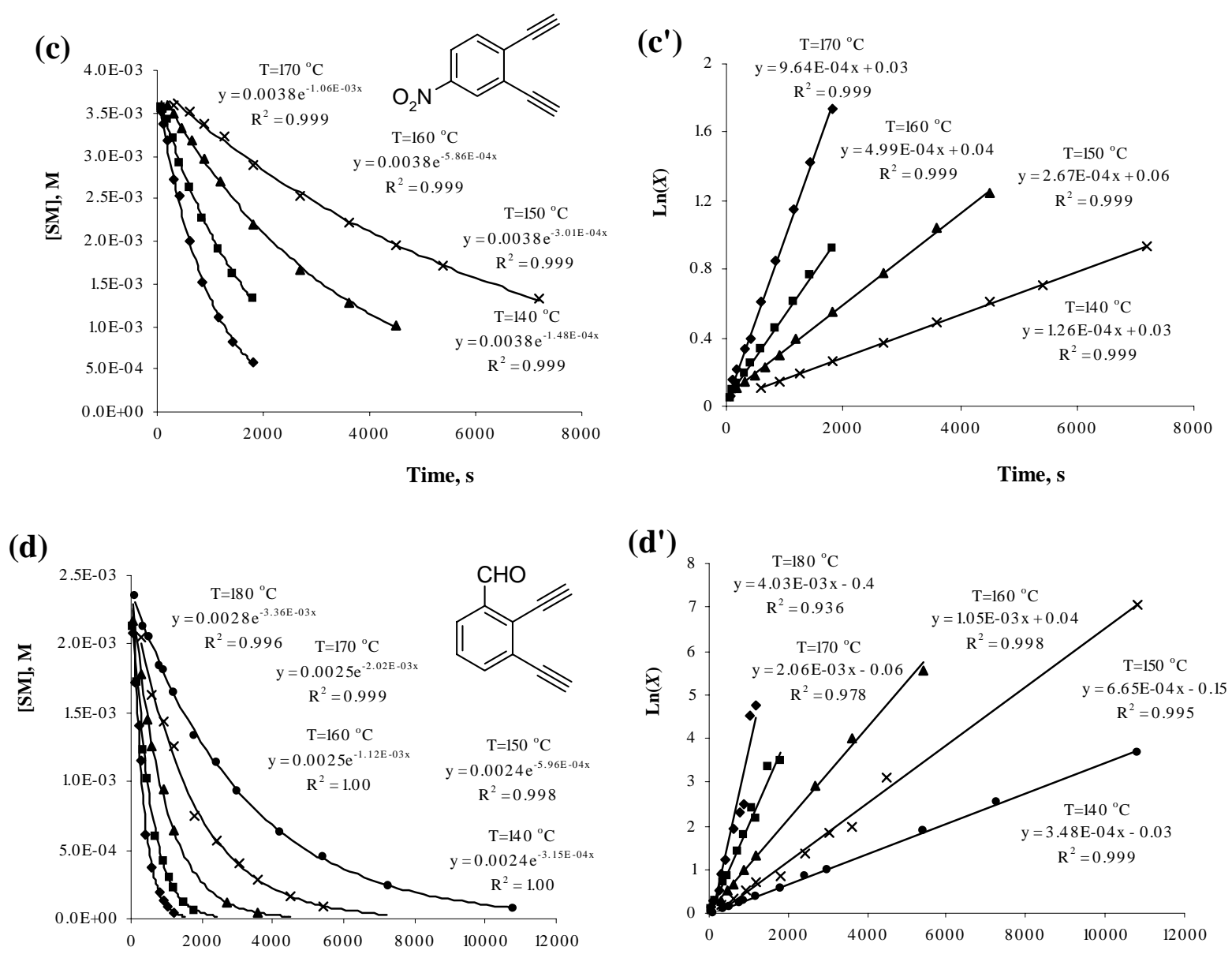

Time, $\mathrm{s}$

Time, $\mathrm{s}$

Figure S1. Continues 
(a)

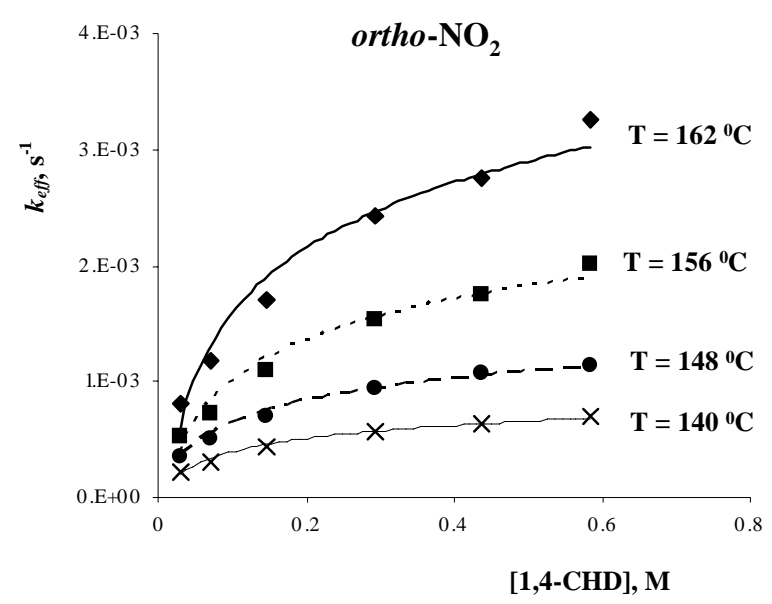

(b)

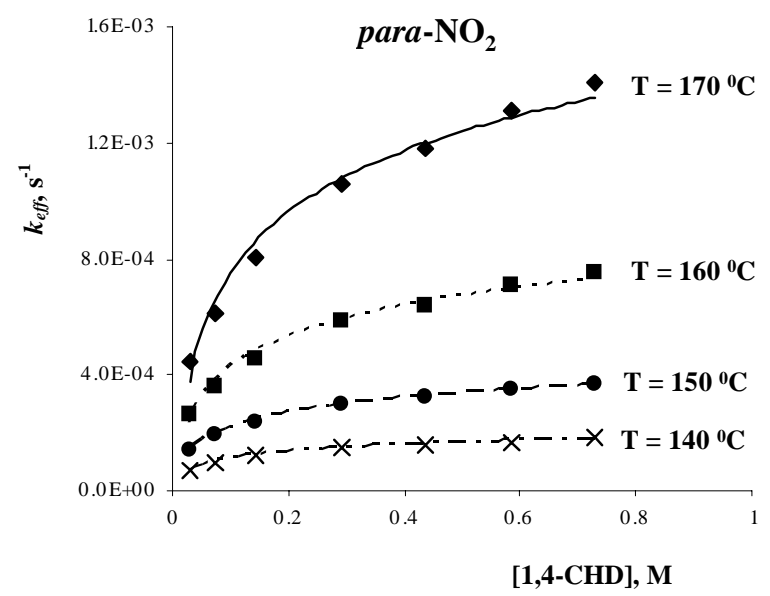

(a')

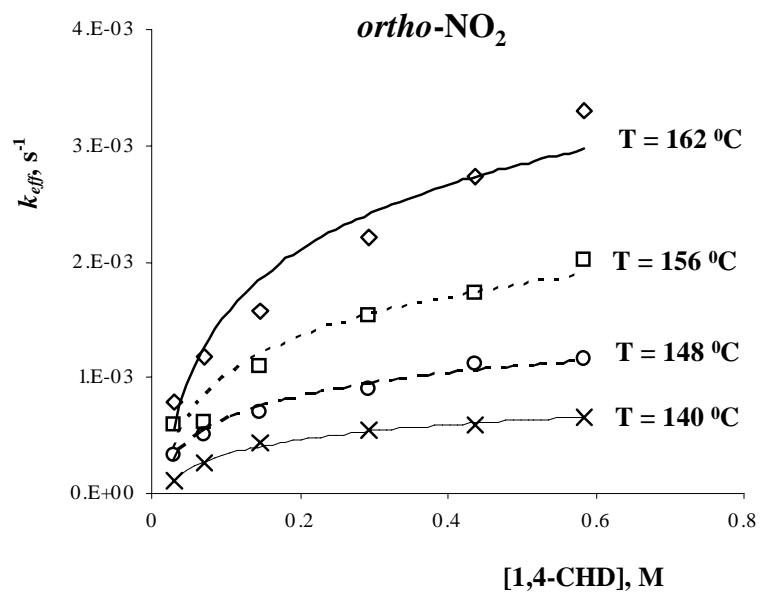

(b')

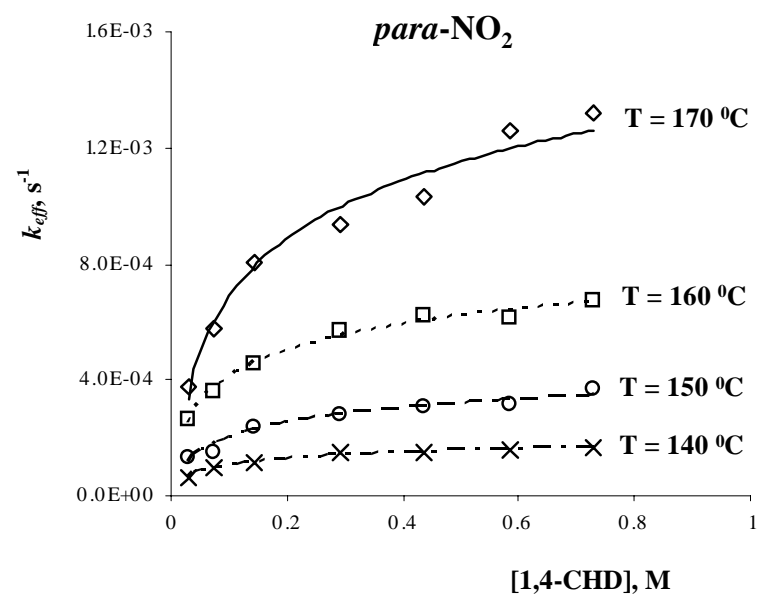

Figure S2. The dependence of effective rate constant, $k_{\text {eff }}$, on 1,4-CHD concentration at different temperatures; (a) and (b) disappearance of ortho- $\mathrm{NO}_{2}$ and para- $\mathrm{NO}_{2}$ enediynes, respectively and (a') and (b') appearance of 1-nitronaphthalenes and 2-nitronapthalene, respectively. 
(a)

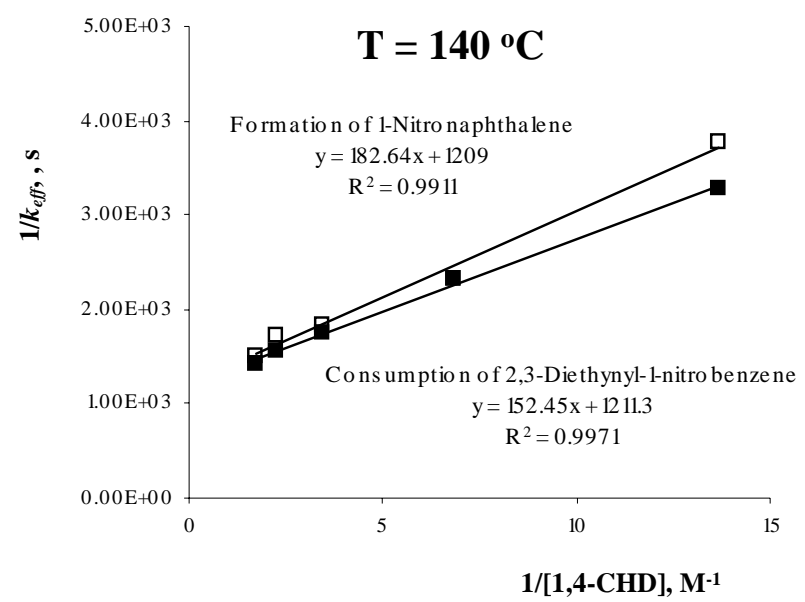

(c)

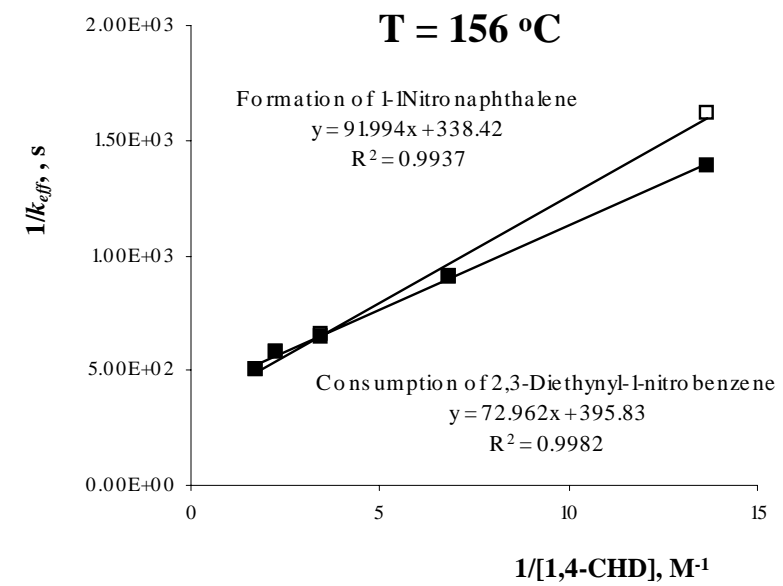

(b)

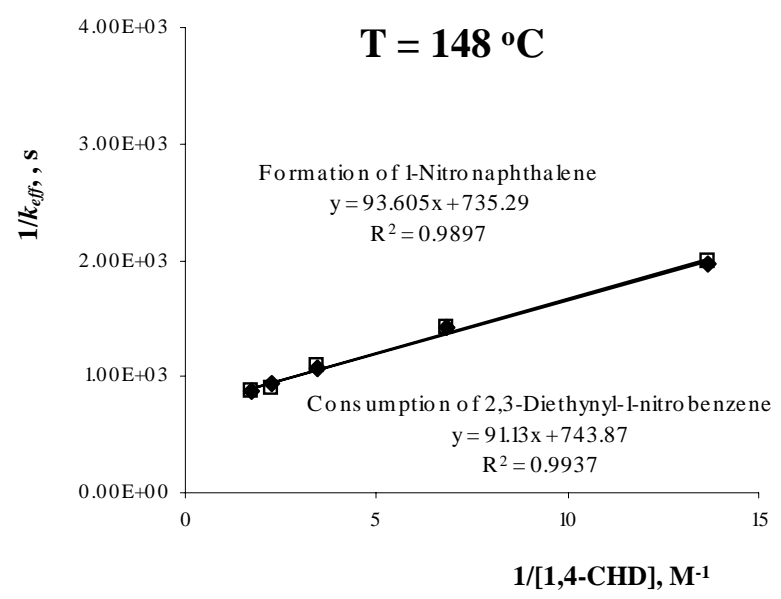

(d)

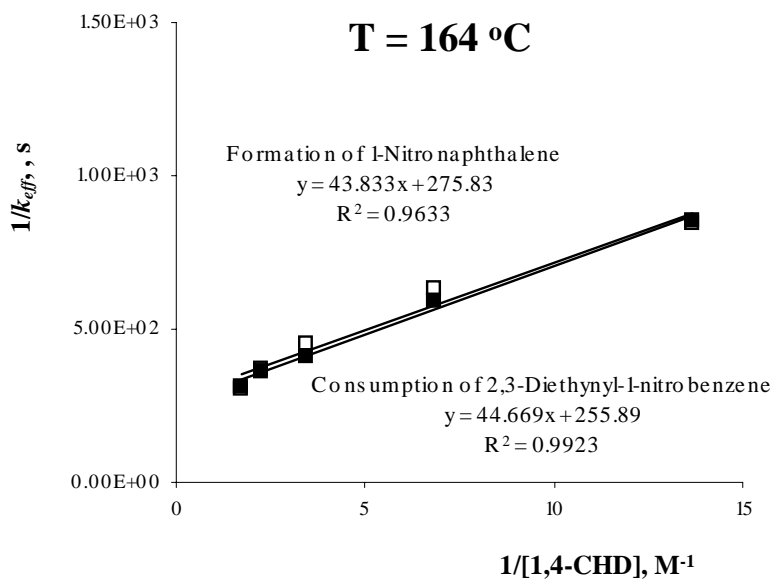

Figure S3. The dependence of effective rate constant, $k_{\text {eff, }}$ on 1,4-CHD concentration for consumption of ortho- $\mathrm{NO}_{2}$ enediyne (filled squares) and formation of 1-nitronaphthalene (hollow squares) at different temperatures using Eq. 6. 
(a)

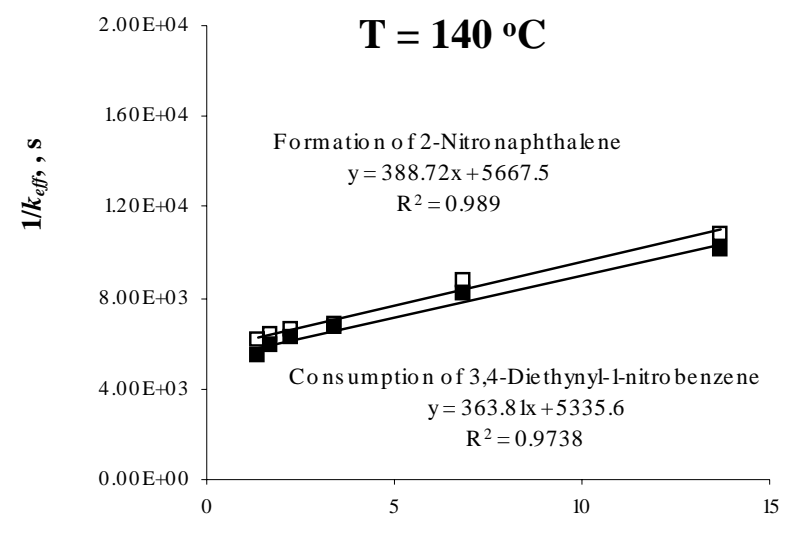

$1 /[1,4-\mathrm{CHD}], \mathrm{M}^{-1}$

(c)

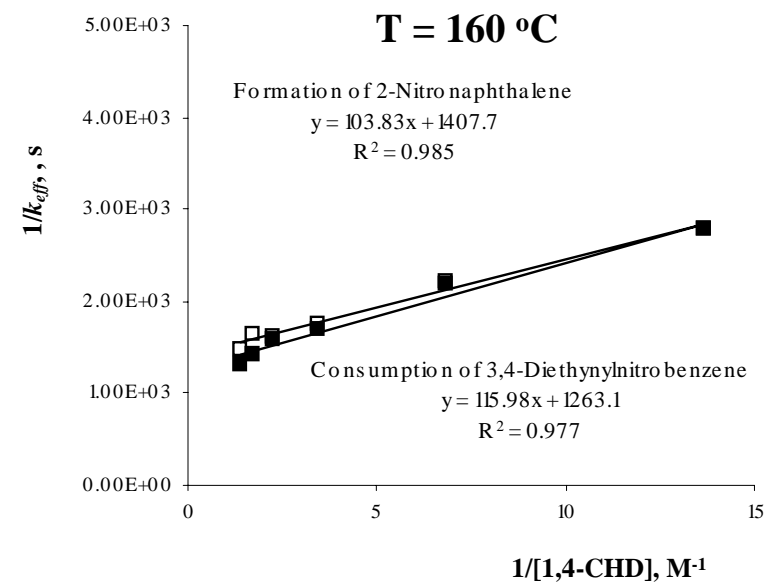

(b)

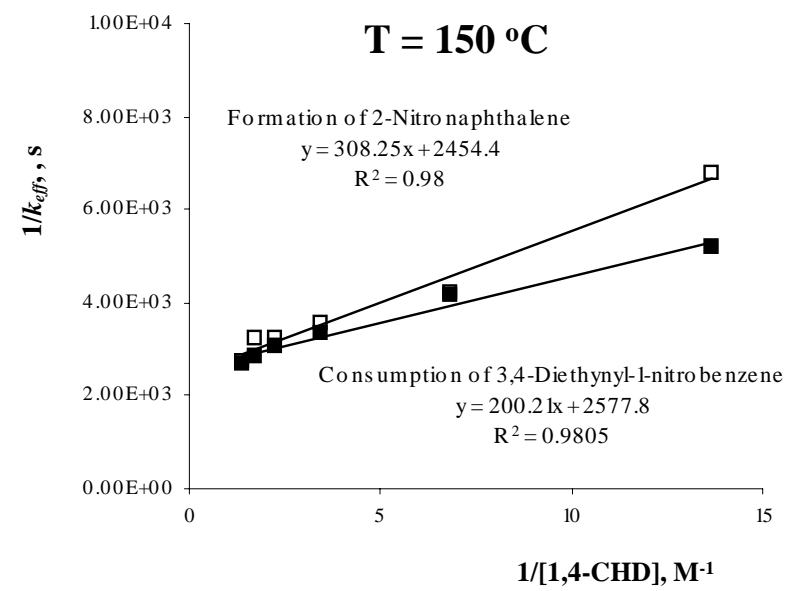

(d)

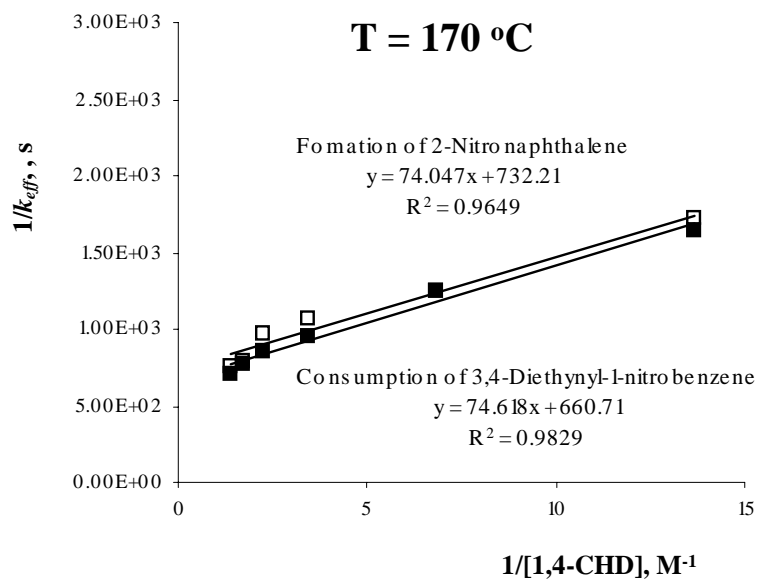

Figure S4. The dependence of effective rate constant, $k_{\text {eff, }}$ on 1,4-CHD concentration for consumption of para- $\mathrm{NO}_{2}$ enediyne (filled squares) and formation of 1-nitronaphthalene (hollow squares) at different temperatures using Eq. 6. 
(a)

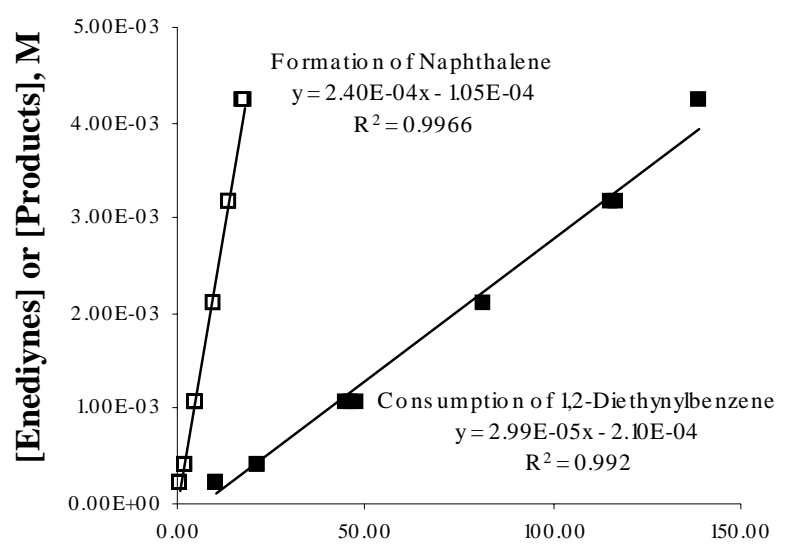

Normalized Area

(c)

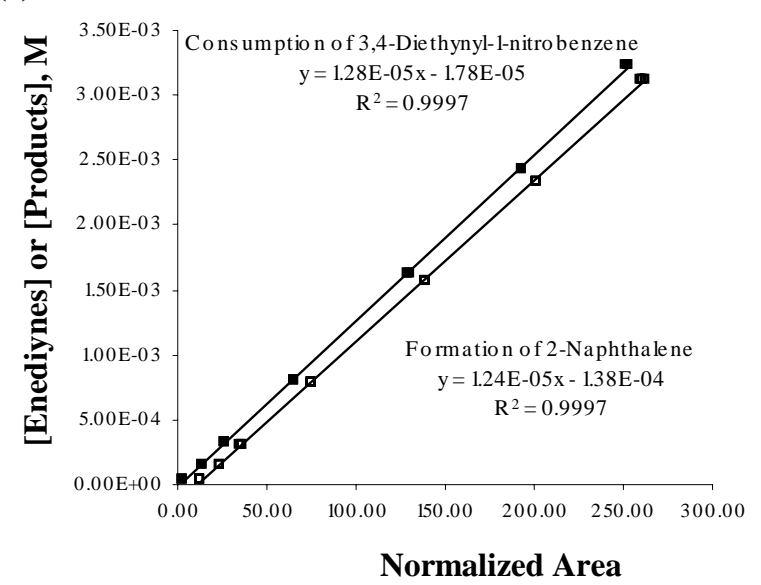

(b)

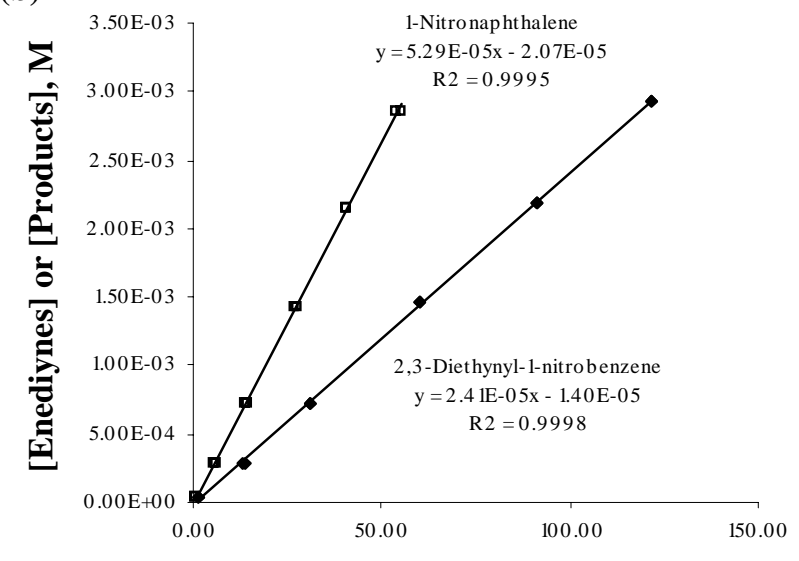

Normalized Area

(d)

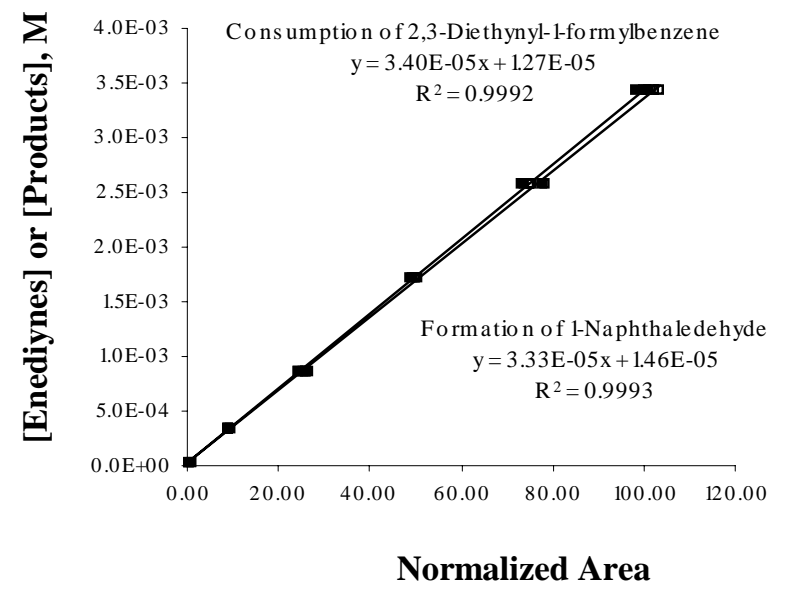

Figure S5. Normalization curves for (a) 1,2-diethynylbenzene 1, (b) 2,3-diethynyl-1nitrobenzene 2, (c) 3,4-diethynyl-1-nitrobenzene 3 and (d) 2,3-diethynyl-1-formyl-beznene 4. 

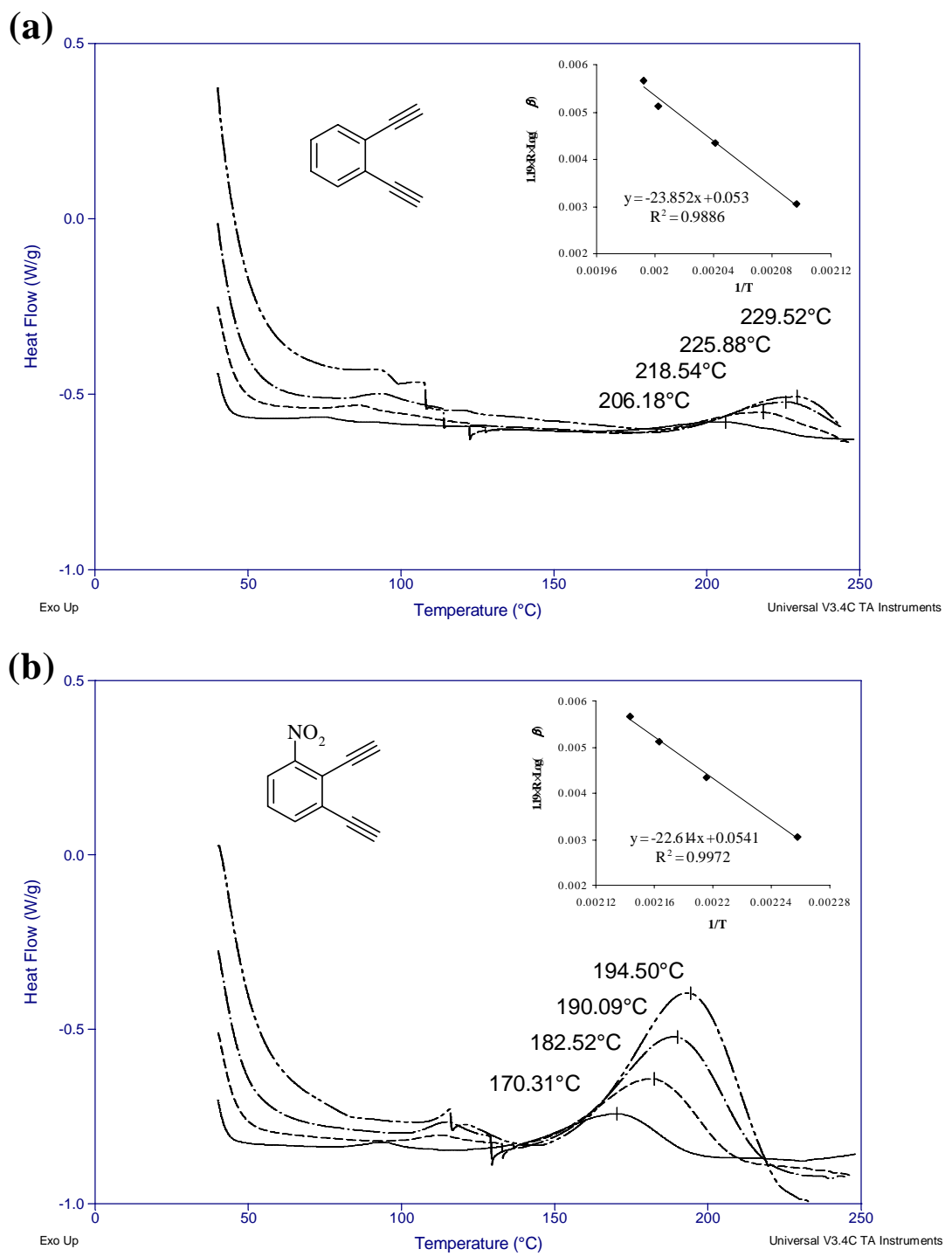

Figure S6. DSC scans for solutions of benzannelated enediynes in 1,4-CHD ([1,4-CHD] $=10.5$ M). Heating rates are 5, 10, 15 and $20^{\circ} \mathrm{C} / \mathrm{min}$ unless noted otherwise. Arrhenius plots are shown in the inset. (a) 1,2-diethynylbenzene, (b) 2,3-diethynylnitrobenzene, (c) 3,4diethynylnitrobenzene (1, 3, 5, $\left.10^{\circ} \mathrm{C} / \mathrm{min}\right)$, (d) 2,3-diethynyl-1-formylbenzene. 
(c)

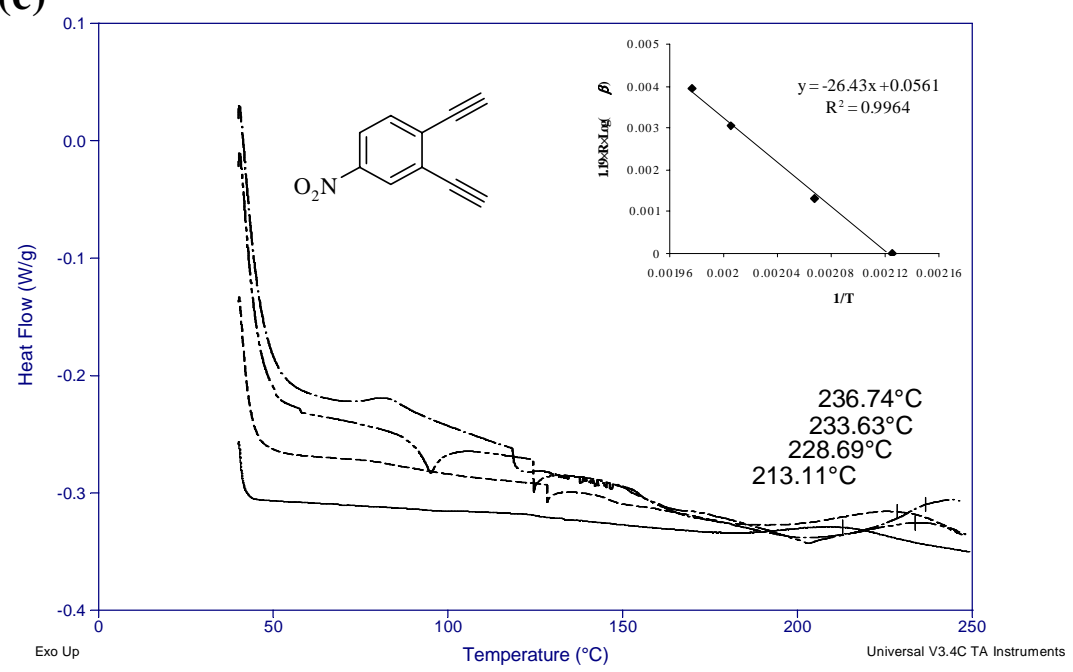

(d)

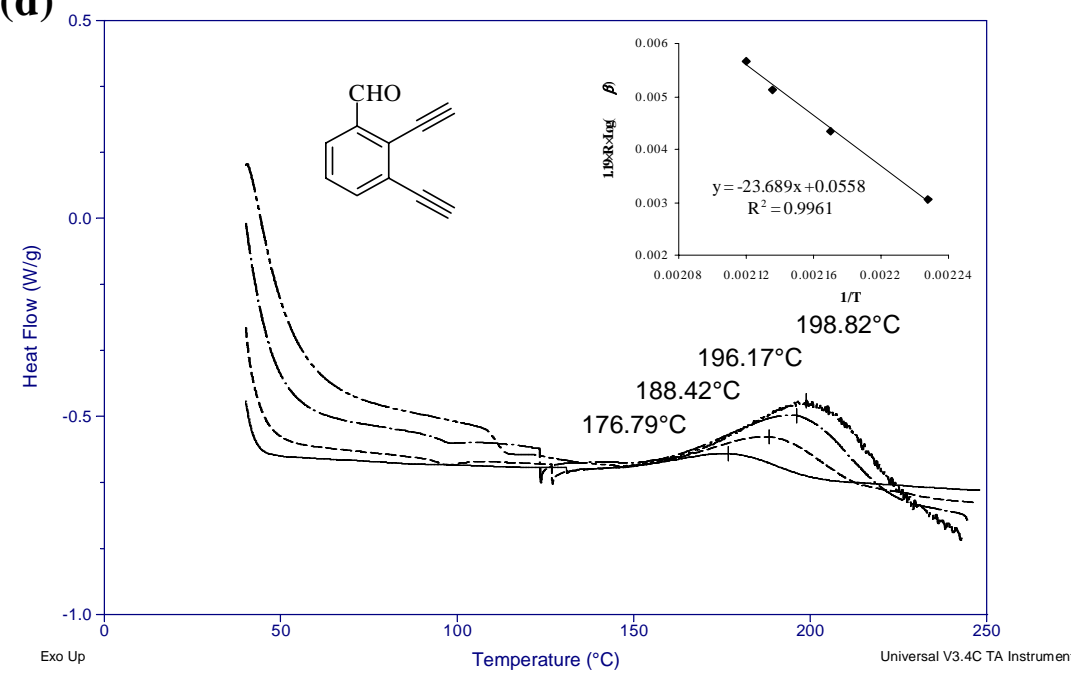

Figure S6. Continues 


\section{Derivations of Equations 1-7:}

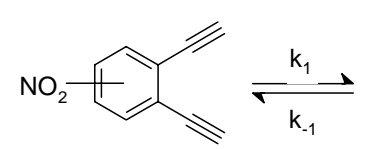

A

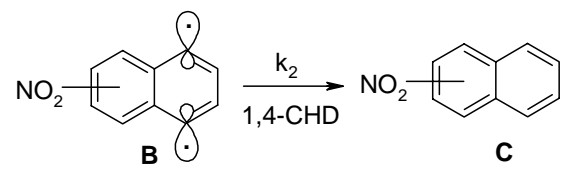

$\mathrm{k}_{3}$

D

(by-products)

$\frac{d[A]}{d t}=-k_{1}[A]+k_{-1}[B]$

A1

$\frac{d[C]}{d t}=k_{2}[H D][B]$

$\frac{d[D]}{d t}=k_{3}[B]$

$\frac{d[B]}{d t}=k_{1}[A]-k_{-1}[B]-k_{3}[B]-k_{2}[H D][B]$

Under Steady State Approximation conditions, $\frac{d[B]}{d t}=0$

So,

$$
k_{1}[A]-k_{-1}[B]-k_{3}[B]-k_{2}[H D][B]=0
$$

Then,

$$
[B]=\frac{k_{1}[A]}{k_{2}[H D]+k_{-1}+k_{3}}
$$

Equation A1 becomes, $\quad \frac{d[A]}{d t}=-k_{1}[A]+\frac{k_{1} k_{-1}[A]}{k_{2}[H D]+k_{-1}+k_{3}}$

This results to, $\quad \frac{d[A]}{d t}=-[A]\left(\frac{k_{1} k_{2}[H D]+k_{1} k_{3}}{k_{2}[H D]+k_{-1}+k_{3}}\right)$

Integration of Equation A6 gives,
$[A]=[A]_{0} e^{\left(-k_{\text {eff }} t\right)}$
Eq. 1 ;
where $k_{\text {eff }}^{A}=\frac{k_{1} k_{2}[H D]+k_{1} k_{3}}{k_{2}[H D]+\left(k_{-1}+k_{3}\right)}$
Eq. 3

If $1,4-\mathrm{CHD}$ is in excess, $k_{2}$ and $k_{-1}$ become more important than $k_{3}$,

Thus, $\quad k_{\text {eff }}=\frac{k_{1} k_{2}[H D]}{k_{2}[H D]+k_{-1}}$

Eq. 5

Substitution of Eq.n A5 in Eq. A2 results, 
$\frac{d[C]}{d t}=[A]\left(\frac{k_{1} k_{2}[H D]}{k_{2}[H D]+k_{-1}+k_{3}}\right)$

Thus, $\quad k_{\text {eff }}^{C}=\frac{k_{1} k_{2}[H D]}{k_{2}[H D]+\left(k_{-1}+k_{3}\right)}$

Eq. 4

If $1,4-\mathrm{CHD}$ is in excess, $k_{2}$ and $k_{-1}$ become more important than $k_{3}$,

Thus $\quad[A]=[A]_{0}-[C]$

A8

Substitution of Eq. A8 in Eq. A7 and integration of E. A7 gives

$\ln (X)=\ln \left(\frac{\left[P_{\mathrm{inf}}\right]}{\left[P_{\mathrm{inf}}\right]-[P]}\right)=k_{\text {eff }} t \quad 2 ; \quad$ where $k_{\text {eff }}=\frac{k_{1} k_{2}[H D]}{k_{2}[H D]+k_{-1}}$

Eq. 5

Substitution of Eq. A5 in Eq. A3 results,

$\frac{d[D]}{d t}=[A]\left(\frac{k_{1} k_{3}}{k_{2}[H D]+k_{-1}+k_{3}}\right)$

Thus, $\quad k_{\text {eff }}^{D}=\frac{k_{1} k_{3}}{k_{2}[H D]+\left(k_{-1}+k_{3}\right)}$

A10

The inverse of Eq. 6 leads $\quad \frac{1}{k_{\text {eff }}}=\frac{k_{-1}}{k_{1} k_{2}[H D]}+\frac{1}{k_{1}}$

Eq. 6

Using Eq. 5 and $\mathbf{A 1 0}$ and Eq. 3 can be written as

$k_{\text {eff }}^{A}=\frac{k_{1} k_{2}[H D]+k_{1} k_{3}}{k_{2}[H D]+\left(k_{-1}+k_{3}\right)}=k_{\text {eff }}^{C}+k_{\text {eff }}^{D}=\frac{k_{\text {eff }}^{C}}{\text { Yield }}$

Eq. 3 
$\begin{array}{ll}t z-1 & 7 z-36 \\ \text { exp1 } & \text { s2put }\end{array}$

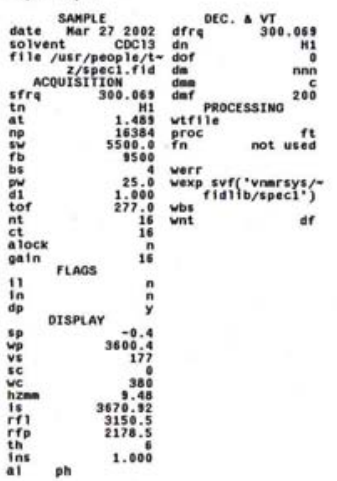
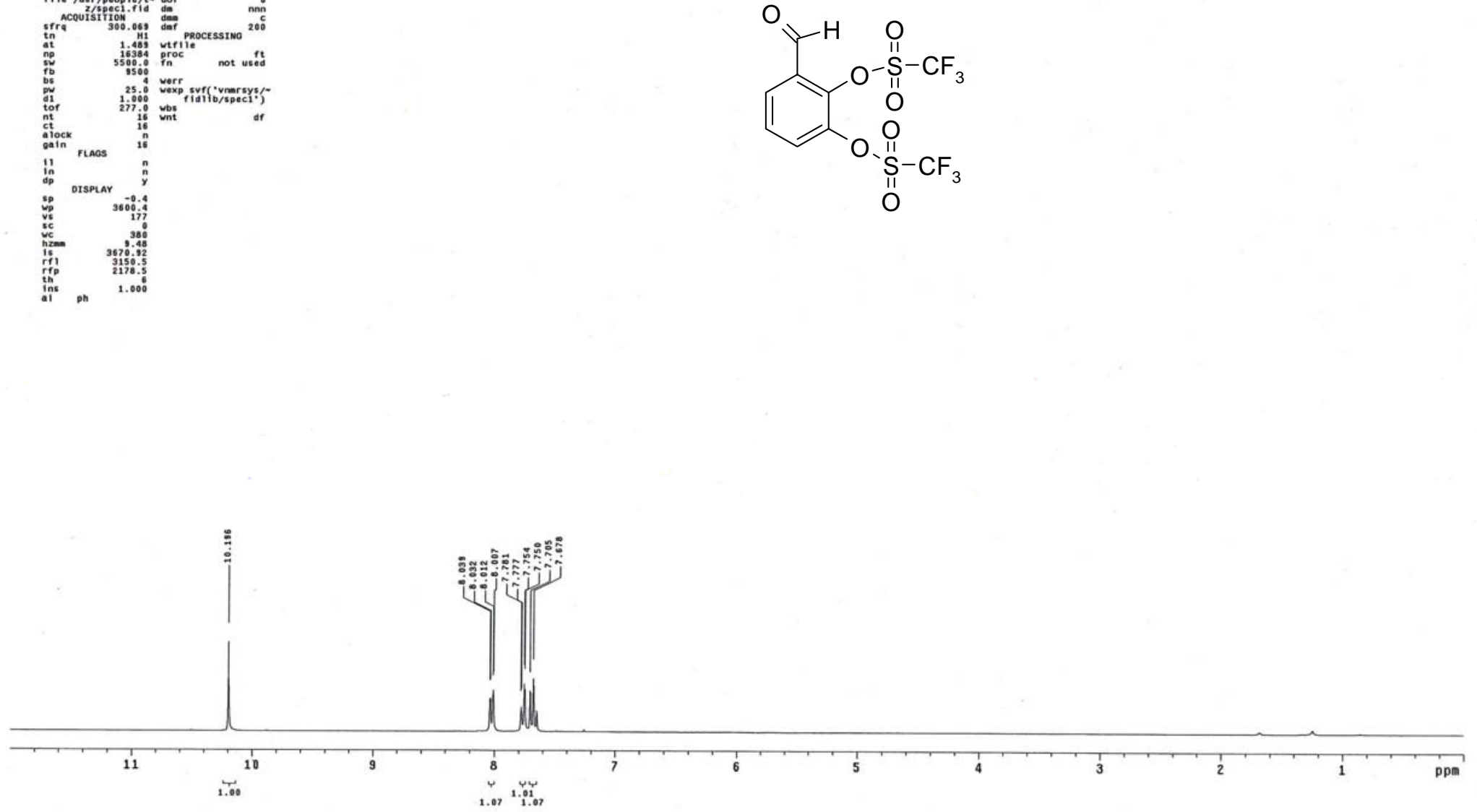


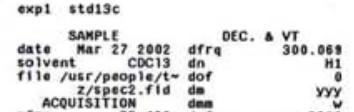

Stra
to
at

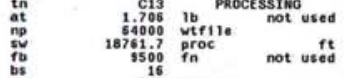

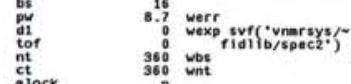

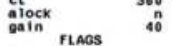

in

ispelar.

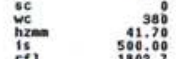

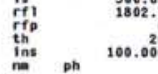

$\overbrace{}^{200}$
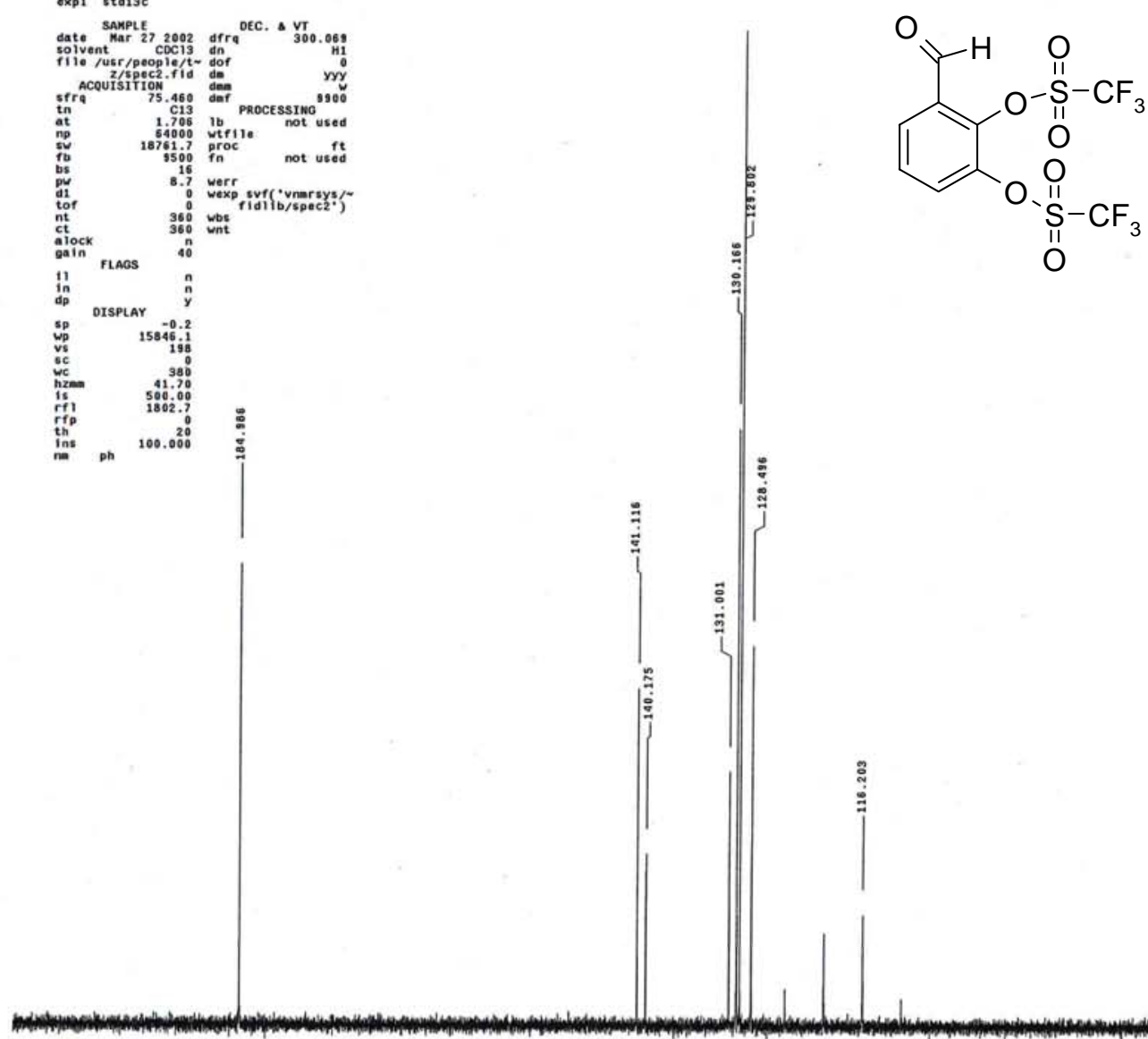
tz-1

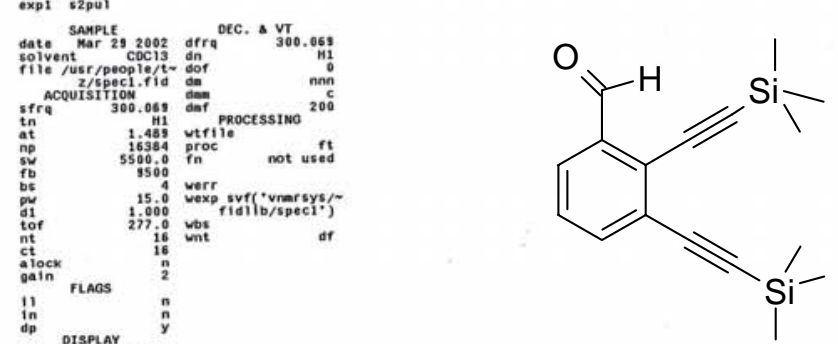

ip 01 splar 3 -300.

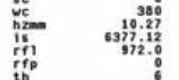

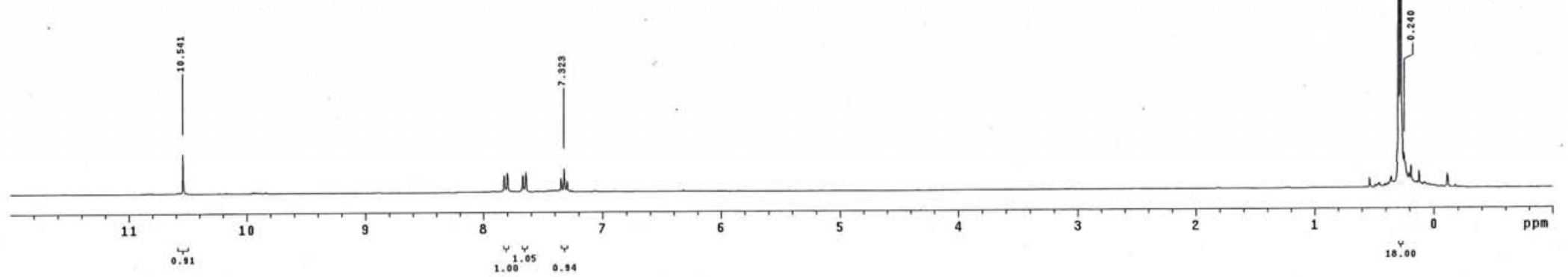


t2-1

exp1 sta13c

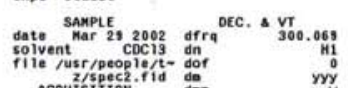

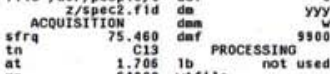

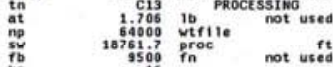

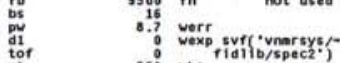

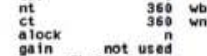

in FlaOS

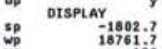

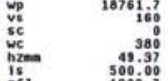

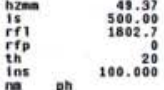
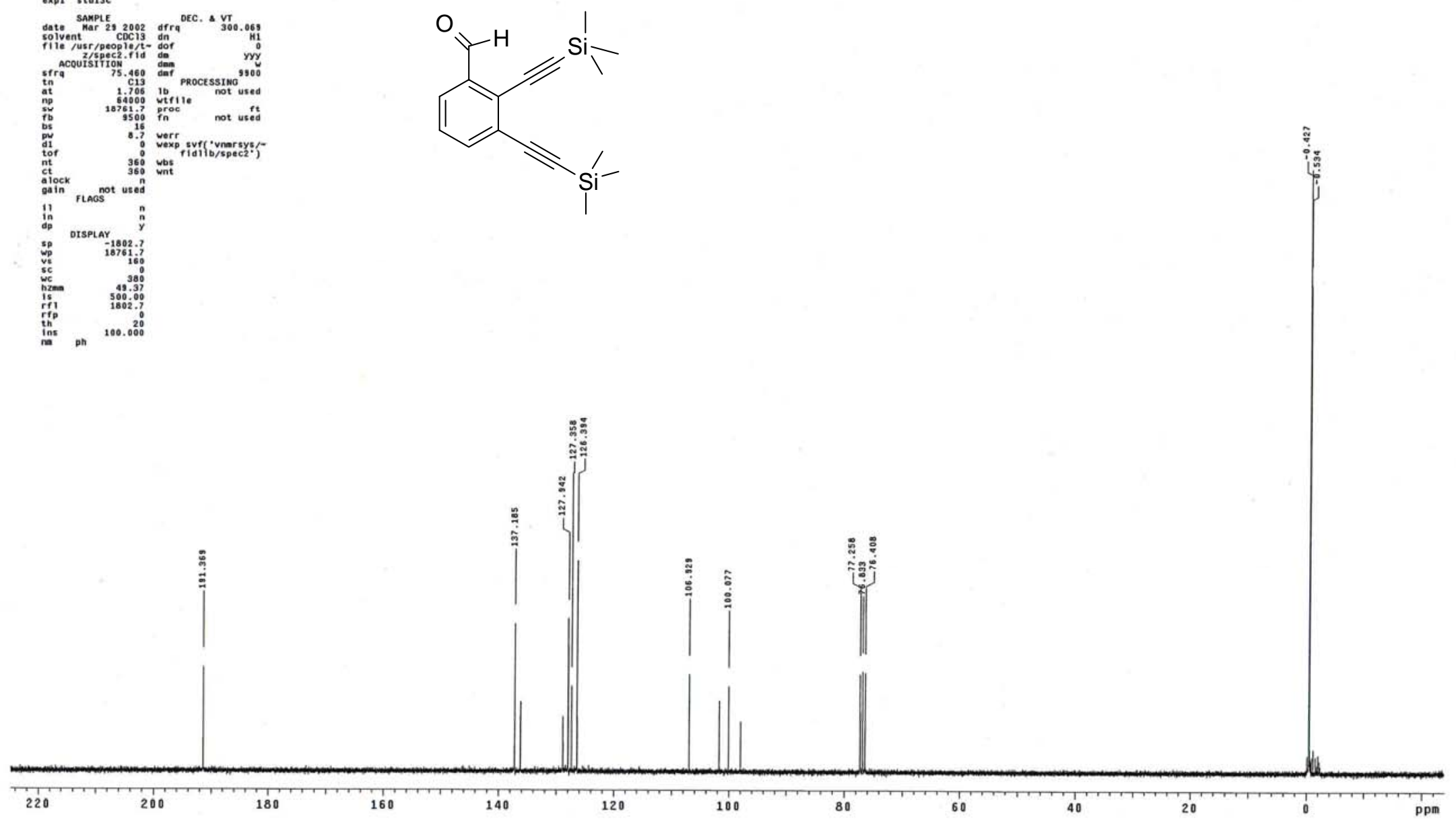


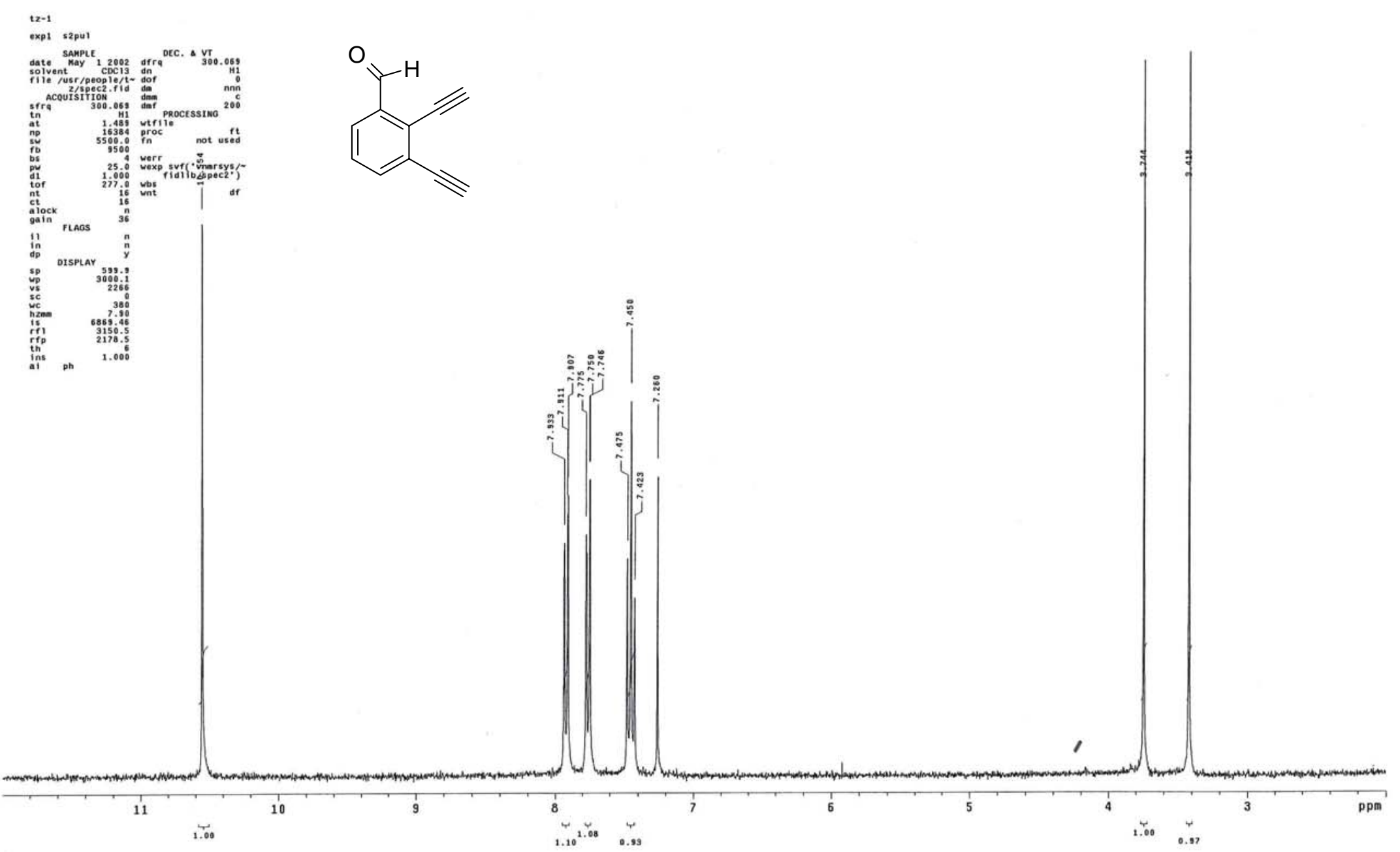


$\begin{array}{ll}t z-1 & 2 \\ \text { exp1 } & \text { sto } \\ & \text { s }\end{array}$
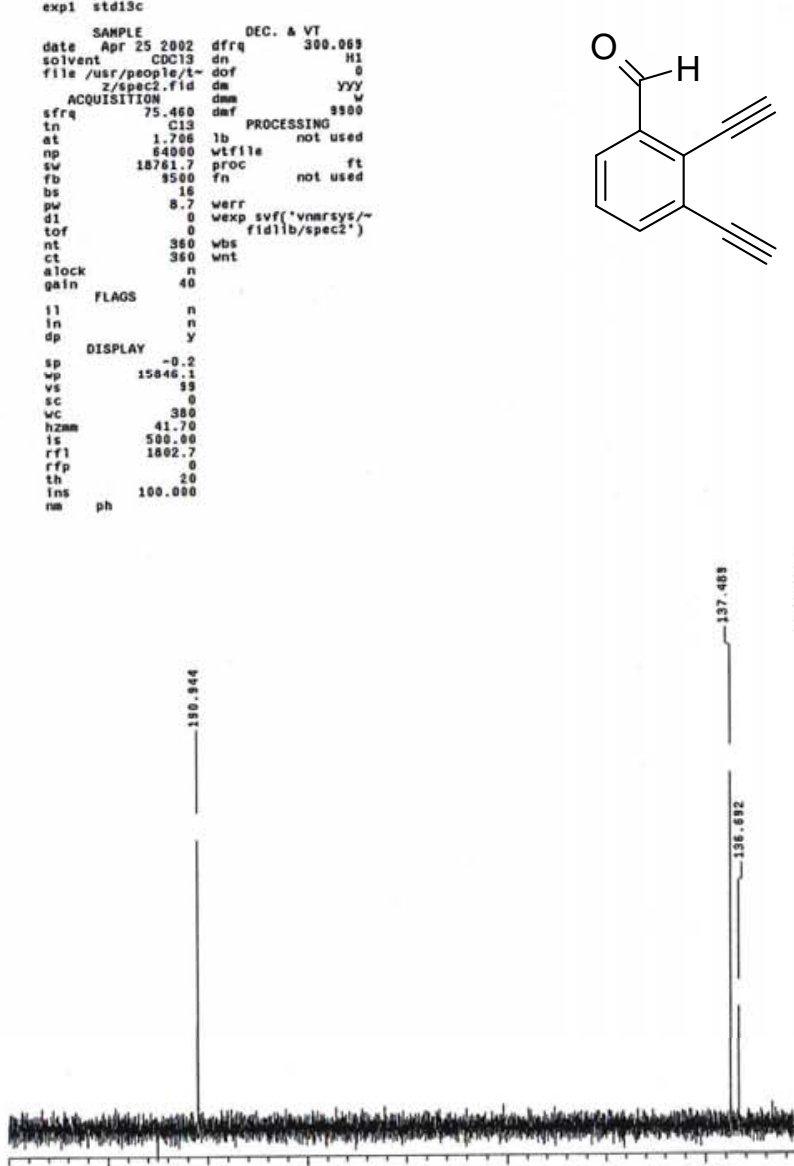
tz-1

oxp1 $52 \mathrm{p}$

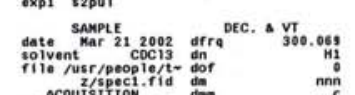

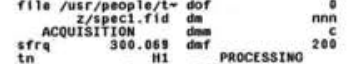

stre
in
at
ng

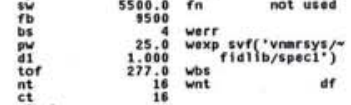

nt
ct lock
gain fla os

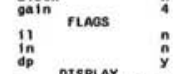

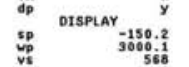

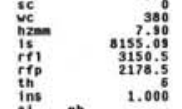
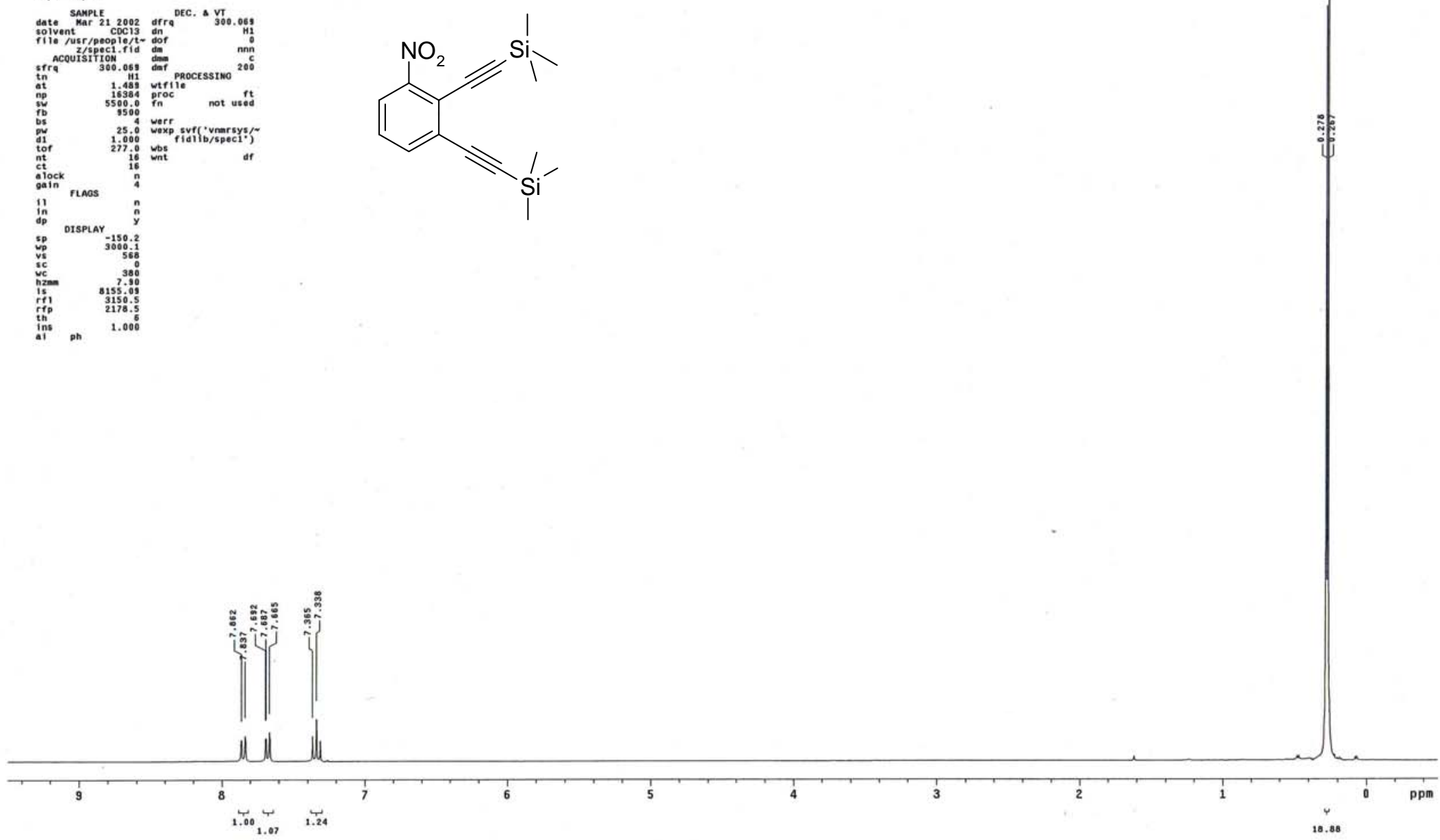
tz-1

$\operatorname{exp1~sta13c}$

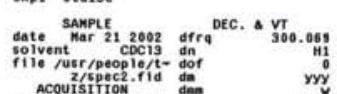

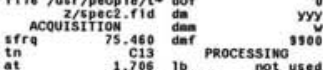

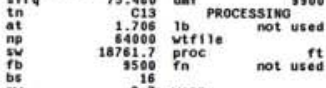

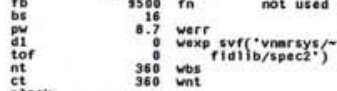

nt
clack
alain
gin

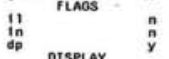

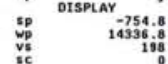

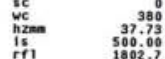

空
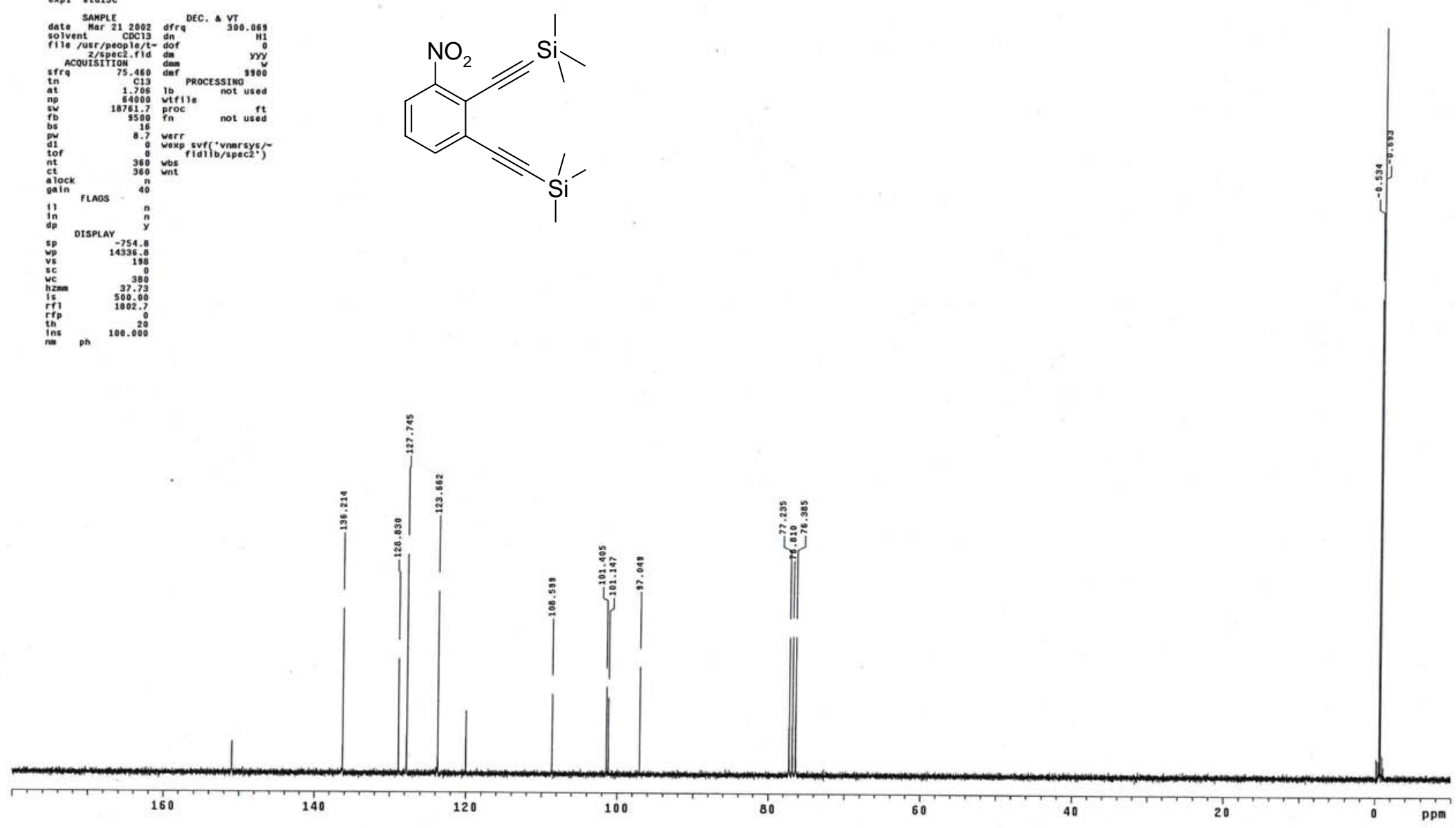
S24
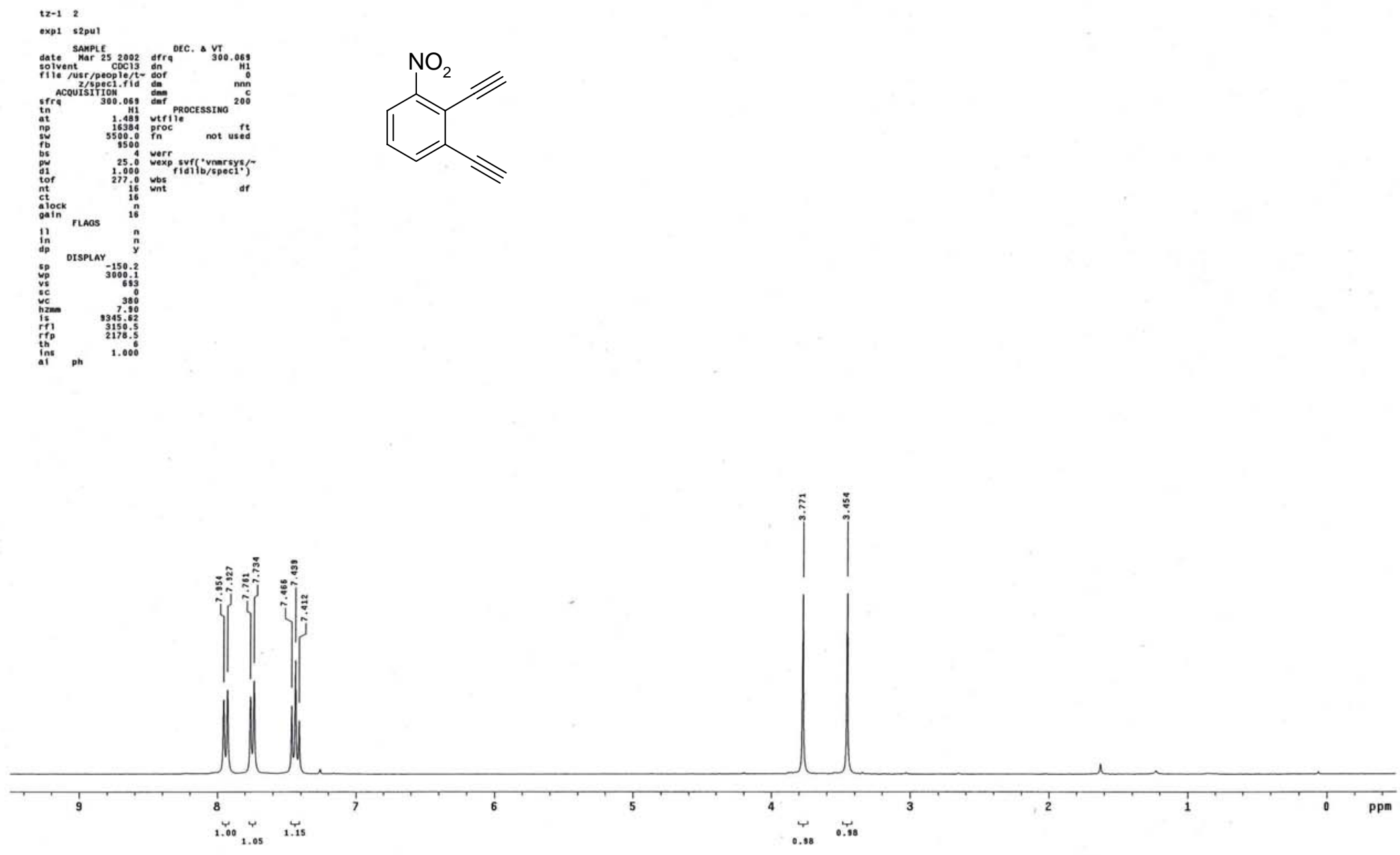


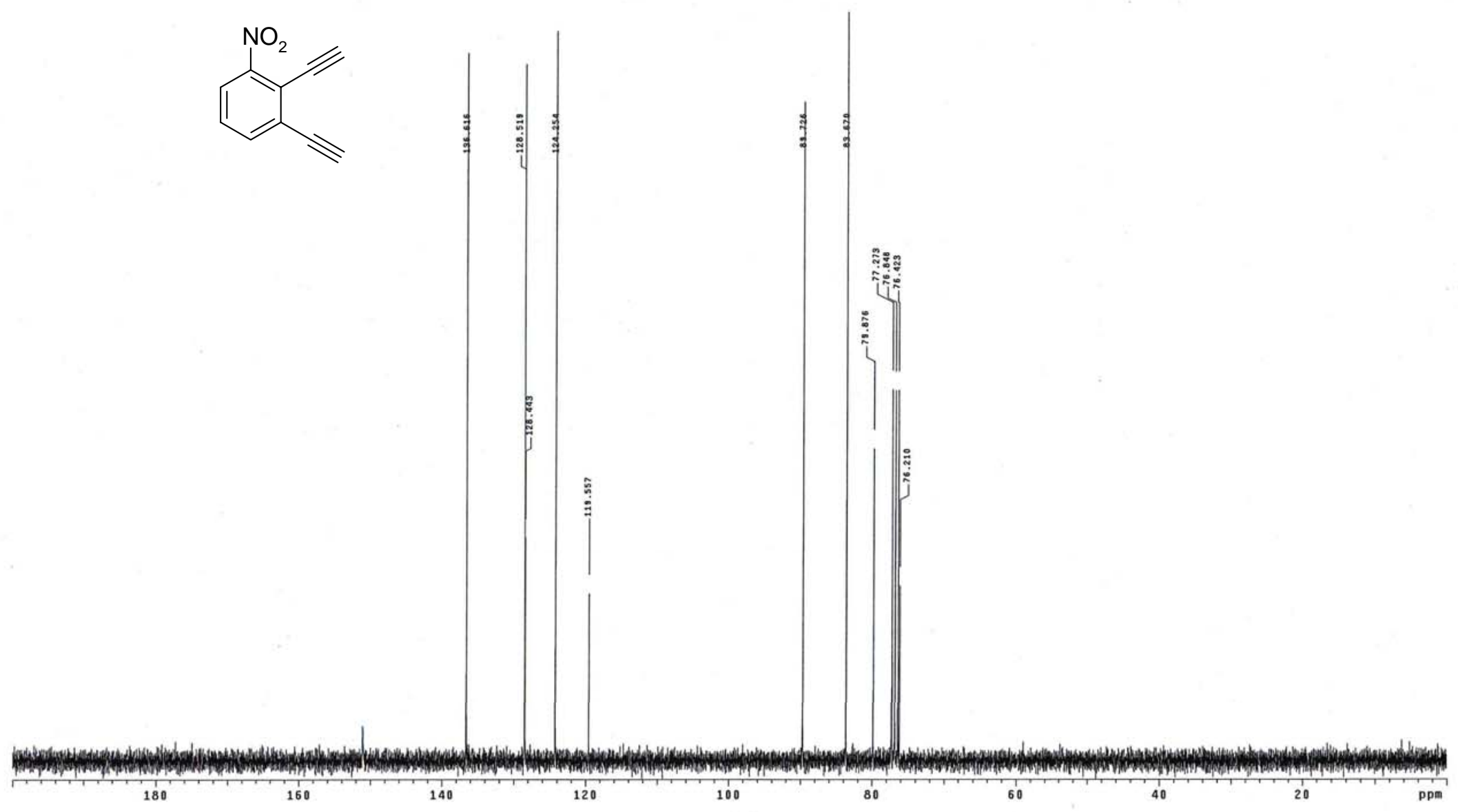


Table S3. The activation barriers for the Bergman cyclizations of various orthosubstituted benzannelated enediynes computed at the B3LYP, BLYP and MP2 levels with both $6-31 \mathrm{G}^{* *}$ and cc-pVDZ basis sets along with the imaginary frequency $\left(\mathrm{cm}^{-1}\right)$ of the TSs.

\begin{tabular}{lcccc}
\hline $\mathrm{X}$ & $\begin{array}{c}\text { BLYP (B3LYP)/ } \\
\text { 6-31G** }\end{array}$ & $\begin{array}{c}\text { BLYP (B3LYP)/ } \\
\text { cc-pVDZ }\end{array}$ & $\begin{array}{c}\text { MP2/ } \\
\text { 6-31G** }\end{array}$ & $\begin{array}{c}\text { Imaginary freq } \\
\text { BLYP (B3LYP)/ } \\
6-31 \mathrm{G}^{* *}\end{array}$ \\
\hline $\mathrm{H}$ & $24.5(31.3)$ & $24.7(31.2)$ & 25.3 & $-682.5(-714.8)$ \\
$\mathrm{NO}_{2}$ & $20.9(27.9)$ & $21.0(27.7)$ & 22.1 & $-438.2(-473.8)$ \\
syn-CHO & $20.6(27.7)$ & $20.7(27.5)$ & 22.1 & $-440.4(-476.3)$ \\
anti-CHO $^{\mathrm{b}}$ & $22.1(29.2)$ & $22.2(29.0)$ & 23.6 & $-442.8(-477.7)$ \\
\hline$p-\mathrm{NO}_{2}$ & $24.1(30.9)$ & $24.2(30.8)$ & 24.9 & $-439.0(-474.3)$ \\
\hline
\end{tabular}

a The oxygen atom $\mathrm{O}$ in $\mathrm{CHO}$ is "syn" to acetylenic group. ${ }^{\mathrm{b}}$ The oxygen atom $\mathrm{O}$ in $\mathrm{CHO}$ is “anti” to acetylenic group. 
Computed stationary point geometries at different levels of theory with total energies (a.u.)

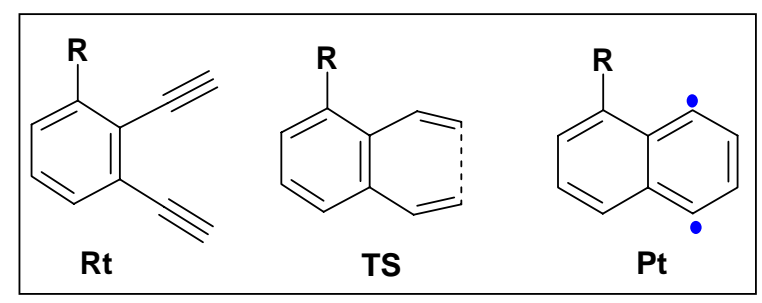

\section{$\mathbf{R t}(\mathbf{R}=\mathbf{H})$}

Total energy $=-384.54661$ (B3L YP/6$31 G^{* *}$ )

$\begin{array}{rrrr}6 & -1.458037 & -1.293284 & 0.000000 \\ 6 & -0.161076 & -0.697087 & 0.000000 \\ 6 & 0.000175 & 0.715193 & 0.000000 \\ 6 & -1.128943 & 1.588491 & 0.000000 \\ 6 & -2.064608 & 2.355502 & 0.000000 \\ 6 & -2.542752 & -1.829168 & 0.000000 \\ 1 & -2.899058 & 3.017565 & 0.000000 \\ 1 & -3.505145 & -2.285724 & 0.000000 \\ 6 & 0.982268 & -1.515053 & 0.000000 \\ 6 & 2.258330 & -0.960924 & 0.000000 \\ 6 & 2.416827 & 0.427255 & 0.000000 \\ 6 & 1.298414 & 1.254570 & 0.000000 \\ 1 & 0.850014 & -2.591745 & 0.000000 \\ 1 & 3.128008 & -1.610732 & 0.000000 \\ 1 & 3.410594 & 0.864272 & 0.000000 \\ 1 & 1.412004 & 2.333392 & 0.000000\end{array}$

Total energy $=-384.64092(\mathrm{BLYP} / 6-$ $31 \mathrm{G} * *)$

$\begin{array}{rrrr}6 & -1.465104 & -1.302303 & 0.000000 \\ 6 & -0.165901 & -0.703871 & 0.000000 \\ 6 & -0.003301 & 0.722931 & 0.000000 \\ 6 & -1.134539 & 1.598342 & 0.000000 \\ 6 & -2.077449 & 2.374258 & 0.000000 \\ 6 & -2.558587 & -1.845832 & 0.000000 \\ 1 & -2.915858 & 3.040730 & 0.000000 \\ 1 & -3.525550 & -2.306379 & 0.000000 \\ 6 & 0.988806 & -1.526247 & 0.000000 \\ 6 & 2.273434 & -0.967325 & 0.000000 \\ 6 & 2.432819 & 0.431247 & 0.000000\end{array}$

$\begin{array}{rrrr}6 & 1.306761 & 1.264646 & 0.000000 \\ 1 & 0.856516 & -2.609941 & 0.000000 \\ 1 & 3.148939 & -1.621160 & 0.000000 \\ 1 & 3.432973 & 0.871313 & 0.000000 \\ 1 & 1.421359 & 2.350357 & 0.000000\end{array}$

Total energy $=-383.34714\left(\mathrm{MP} 2 / 6-31 \mathrm{G}^{* *}\right)$

$6 \begin{array}{llll}6 & -0.555531 & 0.000000 & -1.856478\end{array}$

$\begin{array}{lllll}6 & -0.569245 & 0.000000 & -0.427433\end{array}$

$\begin{array}{llll}6 & 0.642496 & 0.000000 & 0.306512\end{array}$

$\begin{array}{llll}6 & 1.902632 & 0.000000 & -0.367590\end{array}$

$\begin{array}{lllll}6 & 2.993317 & 0.000000 & -0.922333\end{array}$

$\begin{array}{llll}6 & -0.569087 & 0.000000 & -3.080058\end{array}$

$\begin{array}{llll}1 & 3.933615 & 0.000000 & -1.417779\end{array}$

$\begin{array}{llll}1 & -0.565606 & 0.000000 & -4.142892\end{array}$

$\begin{array}{llll}6 & -1.791757 & 0.000000 & 0.263491\end{array}$

$\begin{array}{llll}6 & -1.819780 & 0.000000 & 1.655688\end{array}$

$\begin{array}{llll}6 & -0.624436 & 0.000000 & 2.379700\end{array}$

$\begin{array}{llll}6 & 0.596426 & 0.000000 & 1.710002\end{array}$

$\begin{array}{lllll}1 & -2.712149 & 0.000000 & -0.306445\end{array}$

$\begin{array}{llll}1 & -2.769937 & 0.000000 & 2.174360\end{array}$

$\begin{array}{llll}1 & -0.643992 & 0.000000 & 3.462030\end{array}$

$\begin{array}{llll}1 & 1.527853 & 0.000000 & 2.261718\end{array}$

\section{TS $(\mathbf{R}=\mathbf{H})$}

Total energy $=-384.49676(\mathrm{~B} 3 \mathrm{~L} Y \mathrm{P} / 6-$ $31 \mathrm{G}^{* *}$ )

$\begin{array}{rrrr}6 & 1.342042 & -1.319401 & 0.000000 \\ 6 & 0.062477 & -0.740210 & 0.000000 \\ 6 & -0.000598 & 0.742921 & 0.000000 \\ 6 & 1.225056 & 1.428863 & 0.000000 \\ 6 & 2.437108 & 1.066711 & 0.000000 \\ 6 & 2.518929 & -0.855647 & 0.000000 \\ 1 & 3.458010 & 1.400079 & 0.000000 \\ 1 & 3.564497 & -1.100898 & 0.000000 \\ 6 & -1.150308 & -1.449821 & 0.000000 \\ 6 & -2.378238 & -0.801289 & 0.000000 \\ 6 & -2.437706 & 0.596034 & 0.000000 \\ 6 & -1.269425 & 1.346738 & 0.000000 \\ 1 & -1.107244 & -2.533158 & 0.000000 \\ 1 & -3.293270 & -1.385587 & 0.000000 \\ 1 & -3.399131 & 1.100366 & 0.000000 \\ 1 & -1.318882 & 2.429802 & 0.000000\end{array}$

Total energy $=-384.58538(\mathrm{BLYP} / 6-$ $31 \mathrm{G}^{* *}$ ) 


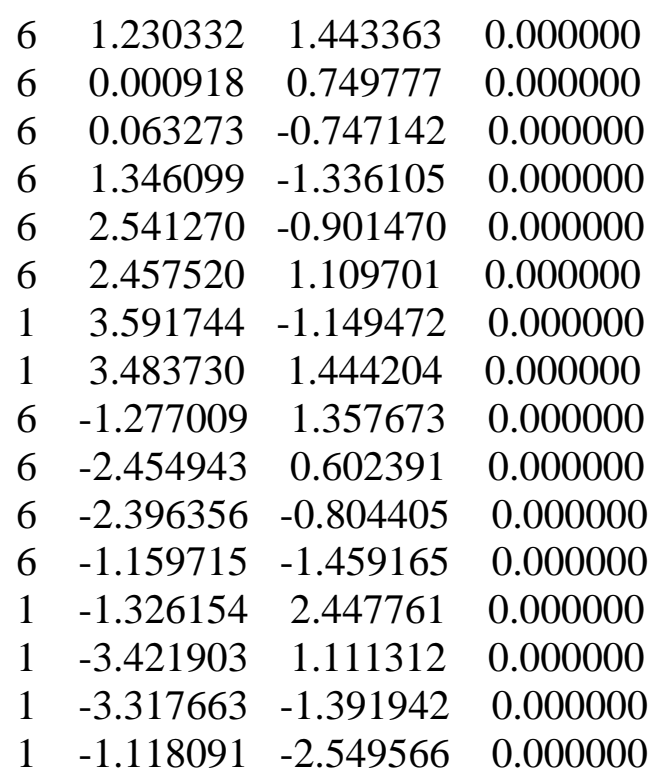

Total energy $=-383.30680\left(\mathrm{MP} 2 / 6-31 \mathrm{G}^{* *}\right)$

$\begin{array}{rrrr}6 & -0.655329 & 0.000000 & -1.762286 \\ 6 & -0.644114 & 0.000000 & -0.355043 \\ 6 & 0.667991 & 0.000000 & 0.307470 \\ 6 & 1.807194 & 0.000000 & -0.518926 \\ 6 & 2.029957 & 0.000000 & -1.772257 \\ 6 & 0.221296 & 0.000000 & -2.685281 \\ 1 & 2.771550 & 0.000000 & -2.545032 \\ 1 & 0.403230 & 0.000000 & -3.740762 \\ 6 & -1.796981 & 0.000000 & 0.453261 \\ 6 & -1.713029 & 0.000000 & 1.837968 \\ 6 & -0.462213 & 0.000000 & 2.469556 \\ 6 & 0.701927 & 0.000000 & 1.715064 \\ 1 & -2.762825 & 0.000000 & -0.034296 \\ 1 & -2.619485 & 0.000000 & 2.429754 \\ 1 & -0.400305 & 0.000000 & 3.550316 \\ 1 & 1.667648 & 0.000000 & 2.202863\end{array}$

\section{Rt $\left(\mathbf{R}=\mathrm{NO}_{2}\right)$}

Total energy $=-589.03509(\mathrm{~B} 3 \mathrm{LYP} / 6-$ $31 G^{* *}$ )

$\begin{array}{llll}6 & -0.833610 & 1.561499 & 0.000000 \\ 6 & 0.000082 & 0.408182 & 0.000000 \\ 6 & 1.414802 & 0.603730 & 0.000000 \\ 6 & 1.972542 & 1.917099 & 0.000000 \\ 6 & 2.497333 & 3.006668 & 0.000000 \\ 6 & -1.419691 & 2.619536 & 0.000000\end{array}$

$\begin{array}{lllc}1 & 2.939811 & 3.976022 & 0.000000 \\ 1 & -1.978817 & 3.526498 & 0.000000 \\ 6 & -0.459031 & -0.927481 & 0.000000 \\ 6 & 0.411959 & -2.014925 & 0.000000 \\ 6 & 1.785506 & -1.800297 & 0.000000 \\ 6 & 2.281056 & -0.500626 & 0.000000 \\ 7 & -1.901937 & -1.245852 & 0.000000 \\ 1 & -0.009882 & -3.010969 & 0.000000 \\ 1 & 2.466106 & -2.644866 & 0.000000 \\ 1 & 3.350079 & -0.318209 & 0.000000 \\ 8 & -2.215688 & -2.437934 & 0.000000 \\ 8 & -2.704240 & -0.318044 & 0.000000\end{array}$

Total energy $=-589.19150(\mathrm{BLYP} / 6-$ 31G**)

$\begin{array}{llll}6 & -0.843105 & 1.564139 & 0.000000 \\ 6 & -0.001836 & 0.413180 & 0.000000 \\ 6 & 1.428258 & 0.613628 & 0.000000 \\ 6 & 1.984478 & 1.930445 & 0.000000 \\ 6 & 2.516544 & 3.029036 & 0.000000 \\ 6 & -1.416558 & 2.642689 & 0.000000 \\ 1 & 2.958420 & 4.005034 & 0.000000 \\ 1 & -1.984024 & 3.551346 & 0.000000 \\ 6 & -0.460195 & -0.935398 & 0.000000 \\ 6 & 0.419113 & -2.029146 & 0.000000 \\ 6 & 1.802071 & -1.810118 & 0.000000 \\ 6 & 2.300559 & -0.500755 & 0.000000 \\ 7 & -1.918639 & -1.257325 & 0.000000 \\ 1 & -0.006454 & -3.031137 & 0.000000 \\ 1 & 2.488735 & -2.658930 & 0.000000 \\ 1 & 3.376321 & -0.316712 & 0.000000 \\ 8 & -2.239034 & -2.468586 & 0.000000 \\ 8 & -2.733279 & -0.313230 & 0.000000\end{array}$

Total energy $=-587.33921\left(\mathrm{MP} 2 / 6-31 \mathrm{G}^{* *}\right)$

$\begin{array}{llll}6 & .241876 & .000000 & -1.745902 \\ 6 & .236497 & .000000 & -.320054 \\ 6 & 1.491984 & .000000 & .351789 \\ 6 & 2.705860 & .000000 & -.400526 \\ 6 & 3.768005 & .000000 & -1.007470 \\ 6 & .421026 & .000000 & -2.956971 \\ 1 & 4.681573 & .000000 & -1.551325 \\ 1 & .507846 & .000000 & -4.016638 \\ 6 & -.915598 & .000000 & .490336 \\ 6 & -.853166 & .000000 & 1.881605 \\ 6 & .389159 & .000000 & 2.510036\end{array}$




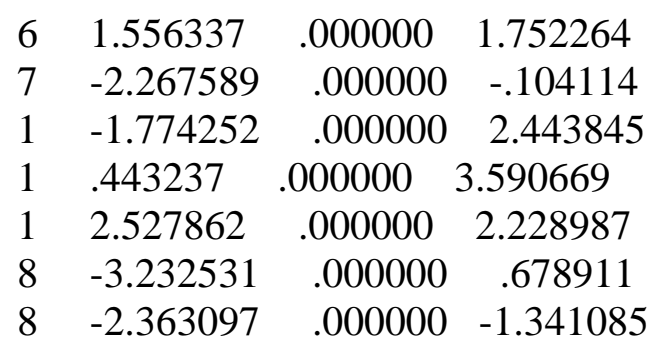

TS $\left(\mathrm{R}=\mathrm{NO}_{2}\right)$

Total energy $=-588.99058(\mathrm{~B} 3 \mathrm{LYP} / 6-$ $\left.31 \mathrm{G}^{* *}\right)$

$\begin{array}{llll}6 & 0.885103 & 1.449148 & 0.000000 \\ 6 & 0.000446 & 0.370664 & 0.000000 \\ 6 & -1.447866 & 0.695611 & 0.000000 \\ 6 & -1.809165 & 2.056146 & 0.000000 \\ 6 & -1.105413 & 3.111645 & 0.000000 \\ 6 & 0.773040 & 2.705783 & 0.000000 \\ 1 & -1.142890 & 4.185976 & 0.000000 \\ 1 & 1.306565 & 3.637677 & 0.000000 \\ 6 & 0.346421 & -0.990536 & 0.000000 \\ 6 & -0.589106 & -2.017258 & 0.000000 \\ 6 & -1.946170 & -1.700621 & 0.000000 \\ 6 & -2.359483 & -0.373458 & 0.000000 \\ 7 & 1.775229 & -1.370047 & 0.000000 \\ 1 & -0.239079 & -3.040960 & 0.000000 \\ 1 & -2.683261 & -2.496452 & 0.000000 \\ 1 & -3.416695 & -0.134676 & 0.000000 \\ 8 & 2.037461 & -2.573444 & 0.000000 \\ 8 & 2.620277 & -0.477055 & 0.000000\end{array}$

Total energy $=-588.84255(\mathrm{BLYP} / 6-$ $31 \mathrm{G} * *)$

$\begin{array}{lllc}6 & 0.898475 & 1.447708 & 0.000000 \\ 6 & -0.004699 & 0.372977 & 0.000000 \\ 6 & -1.463741 & 0.697387 & 0.000000 \\ 6 & -1.830889 & 2.064654 & 0.000000 \\ 6 & -1.160871 & 3.147857 & 0.000000 \\ 6 & 0.822306 & 2.714481 & 0.000000 \\ 1 & -1.198296 & 4.227236 & 0.000000 \\ 1 & 1.362994 & 3.648513 & 0.000000 \\ 6 & 0.344796 & -0.997432 & 0.000000 \\ 6 & -0.595445 & -2.034949 & 0.000000 \\ 6 & -1.961571 & -1.717295 & 0.000000 \\ 6 & -2.380540 & -0.379963 & 0.000000 \\ 7 & 1.790210 & -1.373558 & 0.000000\end{array}$

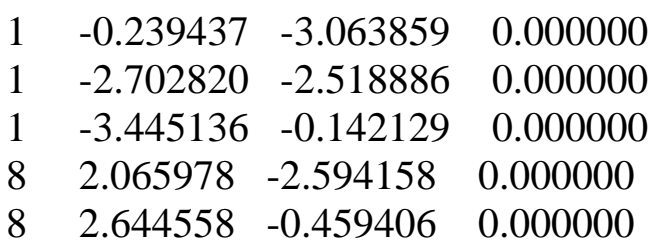
Total energy $=-587.304033\left(\mathrm{MP} 2 / 6-31 \mathrm{G}^{* *}\right)$
$\begin{array}{llll}6 & .203981 & .000000 & -1.705089\end{array}$
$\begin{array}{llll}6 & .231529 & .000000 & -.300641\end{array}$
$\begin{array}{llll}6 & 1.542190 & .000000 & .372725\end{array}$
$\begin{array}{llll}6 & 2.676130 & .000000 & -.457610\end{array}$
$\begin{array}{llll}6 & 2.915220 & .000000 & -1.703730\end{array}$
$\begin{array}{llll}6 & 1.107793 & .000000 & -2.608014\end{array}$
$\begin{array}{llll}1 & 3.667642 & .000000 & -2.466397\end{array}$
$\begin{array}{llll}1 & 1.308032 & .000000 & -3.660481\end{array}$
$\begin{array}{llll}6 & -.893579 & .000000 & .545576\end{array}$
$\begin{array}{llll}6 & -.817715 & .000000 & 1.930556\end{array}$
$\begin{array}{llll}6 & .437999 & .000000 & 2.541647\end{array}$
$\begin{array}{llll}6 & 1.596114 & .000000 & 1.777570\end{array}$
$\begin{array}{lllll}7 & -2.250313 & .000000 & -.045909\end{array}$
$\begin{array}{llll}1 & -1.730320 & .000000 & 2.506461\end{array}$
$\begin{array}{llll}1 & .505353 & .000000 & 3.621572\end{array}$
$\begin{array}{llll}1 & 2.566625 & .000000 & 2.255039\end{array}$
$\begin{array}{llll}8 & -3.210217 & .000000 & .745174\end{array}$
$\begin{array}{llll}8 & -2.360172 & .000000 & -1.281771\end{array}$

\section{Rt $\left(\mathbf{R}=\mathbf{N H}_{2}\right)$}

Total energy $=-439.90786(\mathrm{~B} 3 \mathrm{~L}$ YP/6$31 \mathrm{G}^{* *}$ )

$\begin{array}{llcc}6 & 0.714081 & 1.668293 & 0.000000 \\ 6 & -0.000086 & 0.436213 & 0.000000 \\ 6 & 0.671669 & -0.814176 & 0.000000 \\ 6 & 2.099251 & -0.865383 & 0.000000 \\ 6 & 3.306628 & -0.944537 & 0.000000 \\ 6 & 1.255262 & 2.753082 & 0.000000 \\ 1 & 4.370320 & -0.998385 & 0.000000 \\ 1 & 1.774374 & 3.683200 & 0.000000 \\ 6 & -1.422699 & 0.469637 & 0.000000 \\ 6 & -2.132607 & -0.745204 & 0.000000 \\ 6 & -1.457321 & -1.959395 & 0.000000 \\ 6 & -0.063970 & -2.007957 & 0.000000 \\ 7 & -2.078203 & 1.670371 & 0.000000 \\ 1 & -3.218960 & -0.722394 & 0.000000 \\ 1 & -2.027104 & -2.883945 & 0.000000 \\ 1 & 0.462202 & -2.955173 & 0.000000\end{array}$


$\begin{array}{llll}1 & -3.081540 & 1.711684 & 0.000000\end{array}$

$\begin{array}{llll}1 & -1.553121 & 2.528979 & 0.000000\end{array}$

Total energy $=-439.73183(\mathrm{BLYP} / 6-$ $31 \mathrm{G}^{* *}$ )

$\begin{array}{lccc}6 & 0.718207 & 1.677591 & 0.000000 \\ 6 & 0.004182 & 0.442702 & 0.000000 \\ 6 & 0.681718 & -0.820515 & 0.000000 \\ 6 & 2.111764 & -0.873300 & 0.000000 \\ 6 & 3.330102 & -0.957442 & 0.000000 \\ 6 & 1.254670 & 2.777629 & 0.000000 \\ 1 & 4.399464 & -1.013618 & 0.000000 \\ 1 & 1.777046 & 3.712570 & 0.000000 \\ 6 & -1.432416 & 0.473643 & 0.000000 \\ 6 & -2.147592 & -0.749956 & 0.000000 \\ 6 & -1.467130 & -1.973009 & 0.000000 \\ 6 & -0.064323 & -2.022395 & 0.000000 \\ 7 & -2.090716 & 1.683414 & 0.000000 \\ 1 & -3.241045 & -0.726613 & 0.000000 \\ 1 & -2.040726 & -2.903478 & 0.000000 \\ 1 & 0.464469 & -2.976110 & 0.000000 \\ 1 & -3.101674 & 1.727145 & 0.000000 \\ 1 & -1.557614 & 2.546526 & 0.000000\end{array}$

Total energy $=-438.55269\left(\mathrm{MP} 2 / 6-31 \mathrm{G}^{* *}\right)$

$\begin{array}{lllll}6 & -0.208358 & 0.000000 & -1.791514\end{array}$

$\begin{array}{llll}6 & -0.218932 & 0.000000 & -0.364729\end{array}$

$\begin{array}{llll}6 & 0.985398 & 0.000000 & 0.376834\end{array}$

$\begin{array}{lllll}6 & 2.241047 & 0.000000 & -0.306575\end{array}$

$\begin{array}{lllll}6 & 3.327250 & 0.000000 & -0.871049\end{array}$

$\begin{array}{lllll}6 & -0.281847 & 0.000000 & -3.014694\end{array}$

$\begin{array}{llll}1 & 4.265076 & 0.000000 & -1.370762\end{array}$

$\begin{array}{llll}1 & -0.284882 & 0.000000 & -4.077802\end{array}$

$\begin{array}{llll}6 & -1.464798 & 0.000000 & 0.309907\end{array}$

$\begin{array}{llll}6 & -1.473582 & 0.000000 & 1.714241\end{array}$

$\begin{array}{llll}6 & -0.281799 & 0.000000 & 2.432918\end{array}$

$\begin{array}{llll}6 & 0.948303 & 0.000000 & 1.777820\end{array}$

$\begin{array}{llll}7 & -2.632628 & 0.000000 & -0.408129\end{array}$

$\begin{array}{llll}1 & -2.423933 & 0.000000 & 2.235738\end{array}$

$\begin{array}{llll}1 & -0.315841 & 0.000000 & 3.515243\end{array}$

$\begin{array}{llll}1 & 1.877099 & 0.000000 & 2.332105\end{array}$

$\begin{array}{llll}1 & -3.521005 & 0.000000 & 0.054645\end{array}$

$\begin{array}{llll}1 & -2.604207 & 0.000000 & -1.411220\end{array}$

TS $\left(\mathbf{R}=\mathrm{NH}_{2}\right)$
Total energy $=-439.85642$ (B3L YP/6$31 \mathrm{G}^{* *}$ )

$\begin{array}{llll}6 & 0.919941 & 1.505662 & 0.000000\end{array}$

$\begin{array}{lllll}6 & -0.000055 & 0.446484 & 0.000000\end{array}$

$\begin{array}{llll}6 & 0.539015 & -0.933273 & 0.000000\end{array}$

$\begin{array}{llll}6 & 1.937755 & -1.037458 & 0.000000\end{array}$

$\begin{array}{llll}6 & 2.899539 & -0.216883 & 0.000000\end{array}$

$\begin{array}{llll}6 & 2.187778 & 1.552964 & 0.000000\end{array}$

$\begin{array}{llll}1 & 3.967979 & -0.109948 & 0.000000\end{array}$

$\begin{array}{lllll}1 & 3.046064 & 2.198673 & 0.000000\end{array}$

$\begin{array}{lllll}6 & -1.409643 & 0.623769 & 0.000000\end{array}$

$\begin{array}{lllll}6 & -2.255986 & -0.495367 & 0.000000\end{array}$

$\begin{array}{llll}6 & -1.732128 & -1.785218 & 0.000000\end{array}$

$\begin{array}{llll}6 & -0.360418 & -2.011089 & 0.000000\end{array}$

$\begin{array}{llll}7 & -1.921573 & 1.895939 & 0.000000\end{array}$

$\begin{array}{llll}1 & -3.331961 & -0.343782 & 0.000000\end{array}$

$\begin{array}{lllll}1 & -2.412274 & -2.631906 & 0.000000\end{array}$

$\begin{array}{llll}1 & 0.033347 & -3.019979 & 0.000000\end{array}$

$\begin{array}{lllll}1 & -2.913148 & 2.055886 & 0.000000\end{array}$

$\begin{array}{llll}1 & -1.293780 & 2.681929 & 0.000000\end{array}$

Total energy $=-439.96328(\mathrm{BLYP} / 6-$ $31 \mathrm{G}^{* *}$ )

$\begin{array}{lccc}6 & 0.921379 & 1.518999 & 0.000000 \\ 6 & 0.000688 & 0.449769 & 0.000000 \\ 6 & 0.543290 & -0.939406 & 0.000000 \\ 6 & 1.949797 & -1.053487 & 0.000000 \\ 6 & 2.940441 & -0.257934 & 0.000000 \\ 6 & 2.192767 & 1.607813 & 0.000000 \\ 1 & 4.013865 & -0.148401 & 0.000000 \\ 1 & 3.056131 & 2.255277 & 0.000000 \\ 6 & -1.420083 & 0.628228 & 0.000000 \\ 6 & -2.273927 & -0.499622 & 0.000000 \\ 6 & -1.746214 & -1.797865 & 0.000000 \\ 6 & -0.363656 & -2.024438 & 0.000000 \\ 7 & -1.935216 & 1.909237 & 0.000000 \\ 1 & -3.356810 & -0.346345 & 0.000000 \\ 1 & -2.430325 & -2.650327 & 0.000000 \\ 1 & 0.032619 & -3.039834 & 0.000000 \\ 1 & -2.934159 & 2.072001 & 0.000000 \\ 1 & -1.301693 & 2.700629 & 0.000000\end{array}$

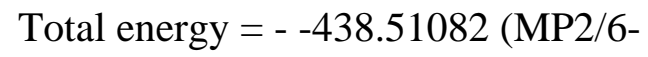
$31 \mathrm{G}^{* *}$ )

$\begin{array}{llll}6 & -0.288785 & 0.000000 & -1.730942\end{array}$

$\begin{array}{lllll}6 & -0.286812 & 0.000000 & -0.324092\end{array}$ 


$\begin{array}{llll}6 & 1.012279 & 0.000000 & 0.353826 \\ 6 & 2.154215 & 0.000000 & -0.468923 \\ 6 & 2.381753 & 0.000000 & -1.723622 \\ 6 & 0.583903 & 0.000000 & -2.653801 \\ 1 & 3.124599 & 0.000000 & -2.495047 \\ 1 & 0.773820 & 0.000000 & -3.707758 \\ 6 & -1.470639 & 0.000000 & 0.455487 \\ 6 & -1.383788 & 0.000000 & 1.850457 \\ 6 & -0.141641 & 0.000000 & 2.488893 \\ 6 & 1.039256 & 0.000000 & 1.760950 \\ 7 & -2.688688 & 0.000000 & -0.181788 \\ 1 & -2.296055 & 0.000000 & 2.435985 \\ 1 & -0.104450 & 0.000000 & 3.571118 \\ 1 & 1.997349 & 0.000000 & 2.261542 \\ 1 & -3.543360 & 0.000000 & 0.341198 \\ 1 & -2.729540 & 0.000000 & -1.183913\end{array}$

\section{Rt (R=syn_CHO)}

Total energy $=-497.86346$ (B3L YP/6$\left.31 \mathrm{G}^{* *}\right)$

$\begin{array}{llcl}6 & 0.140628 & 1.686693 & 0.000000 \\ 6 & 0.000000 & 0.269495 & 0.000000 \\ 6 & 1.171617 & -0.538107 & 0.000000 \\ 6 & 2.473147 & 0.048398 & 0.000000 \\ 6 & 3.594193 & 0.502494 & 0.000000 \\ 6 & 0.349218 & 2.878043 & 0.000000 \\ 1 & 4.573899 & 0.921370 & 0.000000 \\ 1 & 0.491627 & 3.933861 & 0.000000 \\ 6 & -1.264447 & -0.374637 & 0.000000 \\ 6 & -1.339682 & -1.772053 & 0.000000 \\ 6 & -0.190212 & -2.556974 & 0.000000 \\ 6 & 1.055853 & -1.939743 & 0.000000 \\ 6 & -2.555075 & 0.358948 & 0.000000 \\ 1 & -2.320628 & -2.240719 & 0.000000 \\ 1 & -0.262140 & -3.639719 & 0.000000 \\ 1 & 1.964240 & -2.532718 & 0.000000 \\ 1 & -3.438035 & -0.320196 & 0.000000 \\ 8 & -2.702550 & 1.562847 & 0.000000\end{array}$

Total energy $=-497.68893(\mathrm{BLYP} / 6-$ $31 \mathrm{G}^{* *}$ )

$\begin{array}{llll}6 & 0.133451 & 1.695737 & 0.000000\end{array}$

$\begin{array}{llll}6 & -0.003250 & 0.275597 & 0.000000\end{array}$

$\begin{array}{llll}6 & 1.182465 & -0.538636 & 0.000000\end{array}$

$\begin{array}{llll}6 & 2.485609 & 0.050821 & 0.000000\end{array}$

$\begin{array}{llll}6 & 3.618723 & 0.505186 & 0.000000 \\ 6 & 0.366179 & 2.894680 & 0.000000 \\ 1 & 4.602533 & 0.929026 & 0.000000 \\ 1 & 0.506500 & 3.956598 & 0.000000 \\ 6 & -1.277392 & -0.378683 & 0.000000 \\ 6 & -1.349159 & -1.787308 & 0.000000 \\ 6 & -0.189949 & -2.574791 & 0.000000 \\ 6 & 1.064632 & -1.951789 & 0.000000 \\ 6 & -2.573436 & 0.358984 & 0.000000 \\ 1 & -2.336243 & -2.259544 & 0.000000 \\ 1 & -0.259646 & -3.664806 & 0.000000 \\ 1 & 1.979315 & -2.547835 & 0.000000 \\ 1 & -3.463055 & -0.327456 & 0.000000 \\ 8 & -2.722081 & 1.576903 & 0.000000\end{array}$

Total energy $=-496.36928\left(\mathrm{MP} 2 / 6-31 \mathrm{G}^{* *}\right)$

$\begin{array}{llll}6 & -.022498 & .000000 & -1.682262 \\ 6 & -.029537 & .000000 & -.255783 \\ 6 & 1.209798 & .000000 & .434693 \\ 6 & 2.442027 & .000000 & -.288420 \\ 6 & 3.514320 & .000000 & -.877509 \\ 6 & .098673 & .000000 & -2.900380 \\ 1 & 4.436476 & .000000 & -1.406339 \\ 1 & .146374 & .000000 & -3.962322 \\ 6 & -1.223275 & .000000 & .501994 \\ 6 & -1.169931 & .000000 & 1.899802 \\ 6 & .051454 & .000000 & 2.570234 \\ 6 & 1.235900 & .000000 & 1.839261 \\ 6 & -2.574757 & .000000 & -.112220 \\ 1 & -2.098526 & .000000 & 2.460012 \\ 1 & .082209 & .000000 & 3.652096 \\ 1 & 2.195109 & .000000 & 2.341232 \\ 1 & -3.394433 & .000000 & .632478 \\ 8 & -2.820032 & .000000 & -1.311703\end{array}$

\section{TS (R=syn_CHO)}

Total energy $=-497.81927(\mathrm{~B} 3 \mathrm{~L} Y \mathrm{P} / 6-$ $31 \mathrm{G}^{* *}$ )

$\begin{array}{rrrr}6 & 0.331967 & 1.583913 & 0.000000 \\ 6 & 0.000000 & 0.226047 & 0.000000 \\ 6 & 1.123002 & -0.735819 & 0.000000 \\ 6 & 2.431618 & -0.214440 & 0.000000 \\ 6 & 2.865588 & 0.976288 & 0.000000 \\ 6 & 1.401920 & 2.251901 & 0.000000 \\ 1 & 3.747734 & 1.589916 & 0.000000\end{array}$




$\begin{array}{llcc}1 & 1.832353 & 3.235319 & 0.000000 \\ 6 & -1.316114 & -0.286260 & 0.000000 \\ 6 & -1.561022 & -1.659765 & 0.000000 \\ 6 & -0.503427 & -2.568574 & 0.000000 \\ 6 & 0.807815 & -2.107303 & 0.000000 \\ 6 & -2.468354 & 0.643063 & 0.000000 \\ 1 & -2.589279 & -2.012355 & 0.000000 \\ 1 & -0.699445 & -3.635900 & 0.000000 \\ 1 & 1.630509 & -2.813492 & 0.000000 \\ 1 & -3.457602 & 0.136198 & 0.000000 \\ 8 & -2.392778 & 1.855751 & 0.000000\end{array}$

Total energy $=-497.65604(\mathrm{BLYP} / 6-$ $31 G^{* *}$ )

$\begin{array}{cccc}6 & 0.316655 & 1.596733 & 0.000000 \\ 6 & -0.000061 & 0.225938 & 0.000000 \\ 6 & 1.129349 & -0.743106 & 0.000000 \\ 6 & 2.446979 & -0.222497 & 0.000000 \\ 6 & 2.923909 & 0.957169 & 0.000000 \\ 6 & 1.371660 & 2.301650 & 0.000000 \\ 1 & 3.810769 & 1.572983 & 0.000000 \\ 1 & 1.796415 & 3.293560 & 0.000000 \\ 6 & -1.326725 & -0.290503 & 0.000000 \\ 6 & -1.574131 & -1.676156 & 0.000000 \\ 6 & -0.509194 & -2.589477 & 0.000000 \\ 6 & 0.812887 & -2.124196 & 0.000000 \\ 6 & -2.477015 & 0.649054 & 0.000000 \\ 1 & -2.609373 & -2.029908 & 0.000000 \\ 1 & -0.705086 & -3.664011 & 0.000000 \\ 1 & 1.640474 & -2.835548 & 0.000000 \\ 1 & -3.477621 & 0.145554 & 0.000000 \\ 8 & -2.392682 & 1.876213 & 0.000000\end{array}$

Total energy $=-496.33412\left(\mathrm{MP} 2 / 6-31 \mathrm{G}^{* *}\right)$

$\begin{array}{llll}6 & .302194 & 1.606619 & .000000 \\ 6 & .000574 & .233675 & .000000 \\ 6 & 1.112635 & -.725584 & .000000 \\ 6 & 2.412370 & -.189984 & .000000 \\ 6 & 2.939643 & .964560 & .000000 \\ 6 & 1.387377 & 2.280195 & .000000 \\ 1 & 3.849279 & 1.530159 & .000000 \\ 1 & 1.826226 & 3.257357 & .000000 \\ 6 & -1.311329 & -.292968 & .000000 \\ 6 & -1.549641 & -1.665338 & .000000 \\ 6 & -.485198 & -2.570501 & .000000 \\ 6 & .822096 & -2.103735 & .000000\end{array}$

$\begin{array}{llcl}6 & -2.485181 & .612465 & .000000 \\ 1 & -2.573184 & -2.023831 & .000000 \\ 1 & -.675354 & -3.635986 & .000000 \\ 1 & 1.649154 & -2.801418 & .000000 \\ 1 & -3.457849 & .084669 & .000000 \\ 8 & -2.436439 & 1.836578 & .000000\end{array}$

\section{Rt (R=anti_CHO)}

Total energy $=-497.86798$ (B3L YP/6$\begin{array}{llcc}\left.31 G^{* *}\right) & & \\ 6 & -0.212709 & 1.820741 & 0.000000 \\ 6 & 0.000000 & 0.408658 & 0.000000 \\ 6 & 1.326678 & -0.104322 & 0.000000 \\ 6 & 2.455979 & 0.768574 & 0.000000 \\ 6 & 3.435704 & 1.477960 & 0.000000 \\ 6 & -0.379284 & 3.019219 & 0.000000 \\ 1 & 4.289146 & 2.115747 & 0.000000 \\ 1 & -0.516685 & 4.075964 & 0.000000 \\ 6 & -1.084816 & -0.499728 & 0.000000 \\ 6 & -0.851821 & -1.879330 & 0.000000 \\ 6 & 0.446610 & -2.376768 & 0.000000 \\ 6 & 1.525742 & -1.495034 & 0.000000 \\ 6 & -2.491976 & -0.019993 & 0.000000 \\ 1 & -1.714799 & -2.536979 & 0.000000 \\ 1 & 0.623963 & -3.447528 & 0.000000 \\ 1 & 2.543268 & -1.871153 & 0.000000 \\ 8 & -3.453242 & -0.766837 & 0.000000 \\ 1 & -2.619607 & 1.078775 & 0.000000\end{array}$

Total energy $=-497.69278(B L Y P / 6-$ $31 \mathrm{G}^{* *}$ )

$\begin{array}{llll}6 & -0.214343 & 1.830896 & 0.000000 \\ 6 & -0.002199 & 0.416532 & 0.000000 \\ 6 & 1.338901 & -0.101195 & 0.000000 \\ 6 & 2.469716 & 0.773797 & 0.000000 \\ 6 & 3.459582 & 1.488300 & 0.000000 \\ 6 & -0.374369 & 3.041741 & 0.000000 \\ 1 & 4.317469 & 2.129825 & 0.000000 \\ 1 & -0.508148 & 4.104764 & 0.000000 \\ 6 & -1.095289 & -0.503401 & 0.000000 \\ 6 & -0.856158 & -1.893019 & 0.000000 \\ 6 & 0.451669 & -2.392096 & 0.000000 \\ 6 & 1.538194 & -1.503797 & 0.000000 \\ 6 & -2.510374 & -0.023857 & 0.000000 \\ 1 & -1.724883 & -2.554749 & 0.000000\end{array}$




$$
\begin{array}{cccc}
1 & 0.631674 & -3.469645 & 0.000000 \\
1 & 2.562517 & -1.881527 & 0.000000 \\
8 & -3.484325 & -0.777049 & 0.000000 \\
1 & -2.635994 & 1.084327 & 0.000000
\end{array}
$$$$
\text { Total energy }=-496.37366\left(\mathrm{MP} 2 / 6-31 \mathrm{G}^{* *}\right)
$$$$
\begin{array}{llll}
6 & .068921 & .000000 & -1.823468
\end{array}
$$$$
\begin{array}{llll}
6 & .061867 & .000000 & -.393733
\end{array}
$$$$
\begin{array}{llll}
6 & 1.292199 & .000000 & .310990
\end{array}
$$$$
\begin{array}{llll}
6 & 2.533596 & .000000 & -.396345
\end{array}
$$$$
\begin{array}{lllll}
6 & 3.609824 & .000000 & -.978197
\end{array}
$$$$
\begin{array}{llll}
6 & .091010 & .000000 & -3.047558
\end{array}
$$$$
\begin{array}{llll}
1 & 4.537860 & .000000 & -1.496733
\end{array}
$$$$
\begin{array}{llll}
1 & .114171 & .000000 & -4.110670
\end{array}
$$$$
\begin{array}{llll}
6 & -1.145610 & .000000 & .336264
\end{array}
$$$$
\begin{array}{llll}
6 & -1.127314 & .000000 & 1.734543
\end{array}
$$$$
\begin{array}{llll}
6 & .083860 & .000000 & 2.420426
\end{array}
$$$$
\begin{array}{llll}
6 & 1.286749 & .000000 & 1.714135
\end{array}
$$$$
\begin{array}{lllll}
6 & -2.460193 & .000000 & -.356890
\end{array}
$$$$
\begin{array}{llll}
1 & -2.075638 & .000000 & 2.256382
\end{array}
$$$$
\begin{array}{llll}
1 & .096557 & .000000 & 3.502859
\end{array}
$$$$
\begin{array}{llll}
1 & 2.234236 & .000000 & 2.237979
\end{array}
$$$$
\begin{array}{llll}
8 & -3.531627 & .000000 & .243207
\end{array}
$$$$
\begin{array}{llll}
1 & -2.423620 & .000000 & -1.456481
\end{array}
$$

\section{TS (R=anti_CHO)}

Total energy $=-497.82144$ (B3LYP/6-
$\begin{array}{llll}\left.31 G^{* *}\right) \\ 6 & 0.042041 & 1.780011 & 0.000000 \\ 6 & 0.000000 & 0.376557 & 0.000000 \\ 6 & -1.342821 & -0.257212 & 0.000000 \\ 6 & -2.448925 & 0.606502 & 0.000000 \\ 6 & -2.591124 & 1.862114 & 0.000000 \\ 6 & -0.843841 & 2.682493 & 0.000000 \\ 1 & -3.292082 & 2.675670 & 0.000000 \\ 1 & -1.025140 & 3.741283 & 0.000000 \\ 6 & 1.126740 & -0.471126 & 0.000000 \\ 6 & 0.999445 & -1.861401 & 0.000000 \\ 6 & -0.260955 & -2.451947 & 0.000000 \\ 6 & -1.405984 & -1.659848 & 0.000000 \\ 6 & 2.483462 & 0.138169 & 0.000000 \\ 1 & 1.907692 & -2.454931 & 0.000000 \\ 1 & -0.357682 & -3.532963 & 0.000000 \\ 1 & -2.385841 & -2.123943 & 0.000000 \\ 8 & 3.515327 & -0.502522 & 0.000000\end{array}$

\section{$1 \quad 2.482220 \quad 1.249179 \quad 0.000000$}

Total energy $=-497.65750(B L Y P / 6-$ $\begin{array}{llcc}\left.31 \mathrm{G}^{* *}\right) \\ 6 & 0.052105 & 1.788521 & 0.000000 \\ 6 & -0.000834 & 0.377101 & 0.000000 \\ 6 & -1.352862 & -0.259805 & 0.000000 \\ 6 & -2.471060 & 0.602616 & 0.000000 \\ 6 & -2.652901 & 1.859104 & 0.000000 \\ 6 & -0.805403 & 2.726215 & 0.000000 \\ 1 & -3.355880 & 2.677787 & 0.000000 \\ 1 & -0.984277 & 3.790671 & 0.000000 \\ 6 & 1.135219 & -0.477304 & 0.000000 \\ 6 & 1.005662 & -1.879362 & 0.000000 \\ 6 & -0.263408 & -2.471771 & 0.000000 \\ 6 & -1.417097 & -1.672531 & 0.000000 \\ 6 & 2.494778 & 0.141148 & 0.000000 \\ 1 & 1.919449 & -2.477038 & 0.000000 \\ 1 & -0.362423 & -3.559680 & 0.000000 \\ 1 & -2.403463 & -2.139263 & 0.000000 \\ 8 & 3.545295 & -0.494790 & 0.000000 \\ 1 & 2.479033 & 1.262257 & 0.000000\end{array}$

Total energy $=-496.33608\left(\mathrm{MP} 2 / 6-31 \mathrm{G}^{* *}\right)$

$\begin{array}{llll}6 & .043425 & 1.777143 & .000000 \\ 6 & -.005909 & .370852 & .000000 \\ 6 & -1.333745 & -.258586 & .000000 \\ 6 & -2.451960 & .596133 & .000000 \\ 6 & -2.644592 & 1.853525 & .000000 \\ 6 & -.803622 & 2.726522 & .000000 \\ 1 & -3.362400 & 2.648511 & .000000 \\ 1 & -.957987 & 3.786546 & .000000 \\ 6 & 1.125665 & -.472565 & .000000 \\ 6 & 1.004931 & -1.860137 & .000000 \\ 6 & -.258450 & -2.452514 & .000000 \\ 6 & -1.403864 & -1.663681 & .000000 \\ 6 & 2.479666 & .138708 & .000000 \\ 1 & 1.911033 & -2.452467 & .000000 \\ 1 & -.352210 & -3.530904 & .000000 \\ 1 & -2.382621 & -2.124983 & .000000 \\ 8 & 3.518223 & -.512823 & .000000 \\ 1 & 2.489127 & 1.243464 & .000000\end{array}$

\section{Rt (R=p-NO2)}


Total energy $=-589.04442$ (B3L YP/6$31 \mathrm{G}^{* *}$ )

$\begin{array}{llcc}6 & 1.868789 & 1.693526 & 0.000002 \\ 6 & 0.930178 & 0.619527 & 0.000006 \\ 6 & 1.361256 & -0.737446 & 0.000001 \\ 6 & 2.747661 & -1.065850 & -0.000008 \\ 6 & 3.916956 & -1.375728 & -0.000015 \\ 6 & 2.642815 & 2.622484 & -0.000001 \\ 1 & 4.951066 & -1.633835 & -0.000021 \\ 1 & 3.333290 & 3.434303 & -0.000004 \\ 6 & -0.443608 & 0.900352 & 0.000013 \\ 6 & -1.356369 & -0.146474 & 0.000018 \\ 6 & -0.956994 & -1.482511 & 0.000014 \\ 6 & 0.401401 & -1.766942 & 0.000005 \\ 1 & -0.798583 & 1.922318 & 0.000017 \\ 1 & 0.741507 & -2.796308 & 0.000002 \\ 1 & -1.704861 & -2.264857 & 0.000017 \\ 7 & -2.794781 & 0.167357 & 0.000025 \\ 8 & -3.121558 & 1.353431 & -0.000024 \\ 8 & -3.582374 & -0.778274 & -0.000026\end{array}$

Total energy $=-588.88451\left(\mathrm{BLYP} / 6-31 \mathrm{G}^{* *}\right)$

$\begin{array}{llll}6 & 1.881510 & 1.703483 & -0.000001\end{array}$

$\begin{array}{llll}6 & 0.941792 & 0.626952 & 0.000001\end{array}$

$\begin{array}{llll}6 & 1.378292 & -0.744505 & -0.000001\end{array}$

$\begin{array}{lllll}6 & 2.766756 & -1.073514 & -0.000006\end{array}$

$\begin{array}{llll}6 & 3.946555 & -1.388356 & -0.000010\end{array}$

$\begin{array}{llll}6 & 2.661821 & 2.641844 & -0.000001\end{array}$

$\begin{array}{llll}1 & 4.986050 & -1.648351 & -0.000014\end{array}$

$\begin{array}{lllll}1 & 3.355027 & 3.458794 & -0.000004\end{array}$

$\begin{array}{llll}6 & -0.443966 & 0.906610 & 0.000006\end{array}$

$\begin{array}{llll}6 & -1.363092 & -0.147730 & 0.000008\end{array}$

$\begin{array}{llll}6 & -0.959881 & -1.493593 & 0.000006\end{array}$

$\begin{array}{llll}6 & 0.407518 & -1.780432 & 0.000002\end{array}$

$\begin{array}{llll}1 & -0.803203 & 1.934354 & 0.000007\end{array}$

$\begin{array}{llll}1 & 0.749040 & -2.816694 & 0.000000\end{array}$

$\begin{array}{llll}1 & -1.713155 & -2.280190 & 0.000008\end{array}$

$\begin{array}{llll}7 & -2.818070 & 0.169357 & 0.000011\end{array}$

$\begin{array}{llll}8 & -3.150530 & 1.374847 & -0.000006\end{array}$

$\begin{array}{llll}8 & -3.618356 & -0.792092 & -0.000006\end{array}$

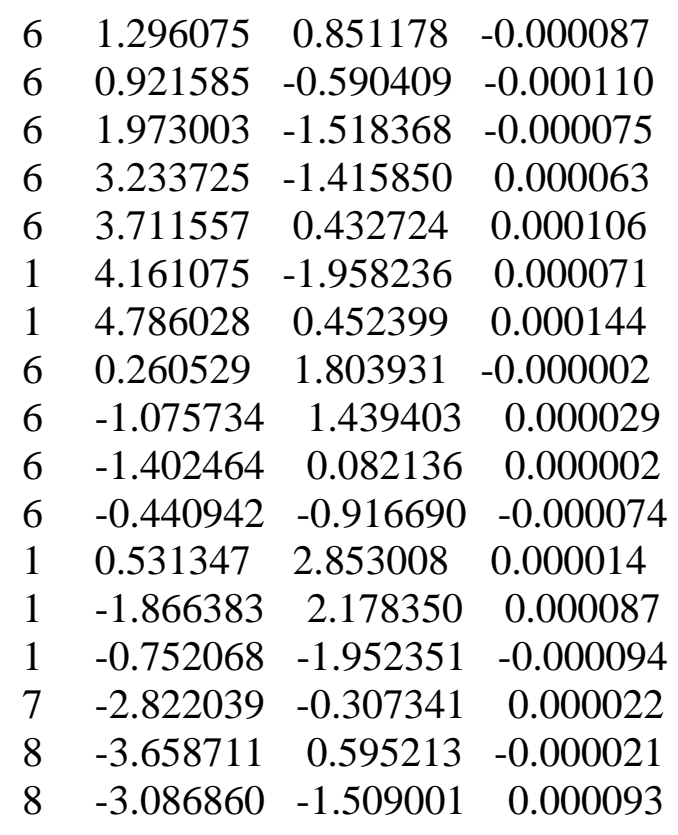

Total energy $=-588.84604(\mathrm{BLYP} / 6-$ $31 \mathrm{G}^{* *}$ )

$\begin{array}{llcc}6 & 2.684336 & 1.162123 & 0.000823 \\ 6 & 1.308469 & 0.858254 & 0.000339 \\ 6 & 0.930626 & -0.594771 & -0.000417 \\ 6 & 1.983732 & -1.533361 & -0.000596 \\ 6 & 3.252976 & -1.469440 & -0.000236 \\ 6 & 3.756777 & 0.477391 & 0.000873 \\ 1 & 4.185346 & -2.013576 & -0.000215 \\ 1 & 4.836478 & 0.496176 & 0.001160 \\ 6 & 0.265121 & 1.817451 & 0.000931 \\ 6 & -1.081364 & 1.450530 & 0.000690 \\ 6 & -1.411234 & 0.083996 & -0.000122 \\ 6 & -0.441389 & -0.922475 & -0.000653 \\ 1 & 0.537511 & 2.873427 & 0.001541 \\ 1 & -1.877674 & 2.193418 & 0.001108 \\ 1 & -0.756723 & -1.964029 & -0.001246 \\ 7 & -2.846004 & -0.309935 & -0.000444 \\ 8 & -3.696696 & 0.607407 & -0.000271 \\ 8 & -3.114704 & -1.531663 & -0.000858\end{array}$

\section{TS (R=p-NO2)}

Total energy $=-588.99503$ (B3L YP/6$31 \mathrm{G}^{* *}$ )

$\begin{array}{llll}6 & 2.665805 & 1.146700 & -0.000010\end{array}$ 